\title{
On Uniform Approximation of Rational Perturbations of Cauchy Integrals
}

\author{
Maxim Yattselev
}

\begin{abstract}
Let $[c, d]$ be an interval on the real line and $\mu$ be a measure of the form $d \mu=\dot{\mu} d \omega_{[c, d]}$ with $\dot{\mu}=h \hbar$, where $\hbar(t)=(t-c)^{\alpha_{c}}(d-t)^{\alpha_{d}}$, $\alpha_{c}, \alpha_{d} \in[0,1 / 2), h$ is a Dini-continuous non-vanishing function on $[c, d]$ with an argument of bounded variation, and $\omega_{[c, d]}$ is the normalized arcsine distribution on $[c, d]$. Further, let $p$ and $q$ be two polynomials such that $\operatorname{deg}(p)<\operatorname{deg}(q)$ and $[c, d] \cap \mathrm{z}(q)=\emptyset$, where $\mathrm{z}(q)$ is the set of the zeros of $q$. We show that AAK-type meromorphic as well as diagonal multipoint Padé approximants to

$$
\mathfrak{f}(z):=\int \frac{d \mu(t)}{z-t}+\left(\frac{p}{q}\right)(z)
$$

converge locally uniformly to $\mathfrak{f}$ in $\mathfrak{D}_{\mathfrak{f}} \cap \mathbb{D}$ and $\mathfrak{D}_{\mathfrak{f}}$, respectively, where $\mathfrak{D}_{\mathfrak{f}}$ is the domain of analyticity of $\mathfrak{f}$ and $\mathbb{D}$ is the unit disk. In the case of Padé approximants we need to assume that the interpolation scheme is "nearly" conjugate-symmetric. A noteworthy feature of this case is that we also allow the density $\dot{\mu}$ to vanish on $(c, d)$, even though in a strictly controlled manner.
\end{abstract}

Mathematics Subject Classification (2000). 42C05, 41A20, 41A21, 41A30.

Keywords. strong asymptotics, non-Hermitian orthogonality, meromorphic approximation, rational approximation, multipoint Padé approximation.

\section{Introduction}

Let $\mathfrak{f}$ be a function of the form

$$
\mathfrak{f}(z):=\int \frac{d \mu(t)}{z-t}+\left(\frac{p}{q}\right)(z), \quad \mathrm{z}(q) \cap[c, d]=\emptyset,
$$

where $[c, d]=\operatorname{supp}(\mu)$ is the support of a complex Borel measure $\mu$, the polynomials $p$ and $q$ are coprime, $\operatorname{deg}(p)<\operatorname{deg}(q)=: m$, and $\mathrm{z}(q)$ is the set of zeros of $q$. Let $\omega_{[c, d]}$ be the equilibrium distribution for $[c, d]$, which is simply the normalized arcsine distribution. In this paper, we assume that $\mu$ is absolutely continuous with 
respect to $\omega_{[c, d]}$ and $\dot{\mu}$, its Radon-Nikodym derivative $\left(d \mu=\dot{\mu} d \omega_{[c, d]}\right)$, is such that

$$
\begin{aligned}
& \dot{\mu}=h \hbar, \\
& \dot{\mu}=h \hbar \hbar_{\mathbf{x}},
\end{aligned}
$$

where $h$ is a non-vanishing Dini-continuous function with argument of bounded variation on $[c, d], \hbar(t):=|t-c|^{\alpha_{c}}|t-d|^{\alpha_{d}}, \alpha_{c}, \alpha_{d} \in[0,1 / 2), \mathbf{x} \subset(c, d)$ is a finite set of distinct points, and $\hbar_{\mathbf{x}}(t):=\prod_{x \in \mathbf{x}}|t-x|^{2 \alpha_{x}}, \alpha_{x} \in(0,1 / 2)$. Under such assumptions on $\mathfrak{f}$, we show locally uniform convergence of $L^{p}(\mathbb{T})$-best meromorphic (in this case we assume that $[c, d] \subset(-1,1)$ ) and certain diagonal multipoint Padé approximants to $\mathfrak{f}$ in

$$
\mathfrak{D}_{\mathfrak{f}}:=\overline{\mathbb{C}} \backslash(\operatorname{supp}(\mu) \cup \mathrm{z}(q)),
$$

the domain of analyticity of $\mathfrak{f}$, where $\overline{\mathbb{C}}$ is the extended complex plane. It is known 25, 5] that the denominators of both types of approximants satisfy non-Hermitian orthogonality relations with respect to $\mu$ that assume a similar form. This leads to similar integral representations for the error of approximation, which is the reason why we treat them simultaneously.

Generally speaking, meromorphic approximants (MAs) are functions meromorphic in the unit disk that provide an optimal approximation to $f$ on the unit circle in the $L^{p}$-norm when the number of poles is fixed. When considering them, it is customary to assume that $\operatorname{supp}(\mu) \cup z(q)$ is contained in the unit disk, $\mathbb{D}$. The study of MAs originated from the work of V.M. Adamyan, D.Z. Arov, and M.G. Krein [1, where the case $p=\infty$ was considered. Nowadays such approximants are often called AAK approximants. The $L^{p}$-extensions of the AAK theory were obtained independently by L. Baratchart and F. Seyfert [5] and V.A. Prokhorov [21]. Meromorphic approximation problems have natural extension to Jordan domains with rectifiable boundary when the approximated function $\mathfrak{f}$ is meromorphic outside of a closed hyperbolic arc of this domain 3 . However, we shall not consider such a generalization here.

The AAK theory itself as well as its generalizations is based on the intimate relation between best (locally best) MAs and Hankel operator whose symbol is the approximated function [1, 5, 21. The study of the asymptotic behavior of MAs is, in fact, equivalent to the study of the asymptotic behavior of the singular vectors and singular numbers of the underlying Hankel operator (see Section 3). Hence, the present work (more specifically, Theorems 1 and 2) can be considered as an asymptotic analysis of the singular vectors of Hankel operators with symbols of type 1.1 $1.2 \mathrm{a}$.

Let us briefly account for the existing results on convergence of MAs to functions of type (1.1). Uniform convergence was obtained in $[\underline{6}$ for the case $p=2$ (in this case meromorphic approximants reduce to rational functions) whenever $\mu$ is a positive measure and the rational summand is not present, i.e. $q \equiv 1$ and necessarily $p \equiv 0$, (such functions $\mathfrak{f}$ are called Markov functions). The general case $p \in[1, \infty]$ was addressed in [4, where again only Markov functions were considered 
and uniform convergence was shown under the assumption that $\mu$ belongs to the $S z e g o$ " class, i.e. $\log (d \mu(t) / d t)$ is integrable on $[c, d]$. The case of complex measures and non-trivial rational part was taken up in [9], where convergence in capacity in $\mathbb{D} \backslash \operatorname{supp}(\mu)$ was obtained while $\operatorname{supp}(\mu)$ was assumed to be a regular set with respect to the Dirichlet problem and $\mu$ had to be sufficiently "thick" on its support and have an argument of bounded variation.

On the other hand, diagonal multipoint Padé approximants (PAs) are rational functions of type $(n, n)$ that interpolate $\mathfrak{f}$ in a system of $2 n$ not necessarily distinct nor finite points (interpolation scheme) lying in $\mathfrak{D}_{\mathfrak{f}}$ with one additional interpolation condition at infinity. Unlike the meromorphic case, it is pointless to assume that $\operatorname{supp}(\mu)$ and $\mathrm{z}(q)$ lie in $\mathbb{D}$. It is customary to call PA classical if all the interpolation points lie at infinity. Such approximants were initially studied by A.A. Markov [17] using the language of continued fractions. Later, A.A. Gonchar [13. considered classical PAs to functions of type (1.1) with nontrivial rational part and positive $\mu$. Locally uniform convergence to $\mathfrak{f}$ in $\mathfrak{D}_{\mathfrak{f}}$ was obtained under the condition that $\mu$ belongs to the Szegö class. Continuing this work, E.A. Rakhmanov has shown 22] that the restriction on $\mu$ to be in the Szego" class cannot be relaxed in general, but if all the coefficients of $R$ are real, uniform convergence holds for any positive measure. In the recent paper [14] A.A. Gonchar and S.P. Suetin proved that uniform convergence of classical PAs still holds if $\mu$ is a complex measure of the form $d \mu=h d \omega_{[c, d]}$, where $h$ is a non-vanishing analytic function in some neighborhood of $[c, d]$. Recently, using the operator-theoretic approach, M.S. Derevyagin and V.A. Derkach [11 showed that there always exists a subsequence of diagonal PAs that converges locally uniformly to $\mathfrak{f}$ whenever the latter is such that $\mu$ is a positive measure and $p / q$ is real-valued on $\operatorname{supp}(\mu)$ but can have poles there. Finally, we mention a weaker result that holds for a larger class of complex measures. It was shown in [9, Thm. 2.3] that multipoint Padé approximants corresponding to "nearly" conjugate-symmetric interpolation schemes converge in capacity in $\mathbb{C} \backslash \operatorname{supp}(\mu)$ whenever $\operatorname{supp}(\mu)$ is a regular set with respect to the Dirichlet problem and $\mu$ is sufficiently "thick" on its support and has an argument of bounded variation.

The main results of this paper are presented in Section 3 , Theorems 14 , and Section 4. Theorems 5 and 6. The conditions imposed on the measure $\mu$ in these theorems come from Theorem 7. The latter is, in fact, a consequence of Theorems 2 and 3 in [7]. In particular, if Theorem 7 is established under other assumptions on $\mu$, this would yield Theorems 16 for this new class of measures. For instance, all the main results of the present work would hold whenever $\mu$ is of the form

$$
d \mu(t)=h(t)(t-c)^{\alpha_{c}}(d-t)^{\alpha_{d}} d t
$$

where $h$ is an $m$-times continuously differentiable non-vanishing function on $[c, d]$ with $m$-th derivative being $\varsigma$-Hölder continuous and $\alpha_{c}, \alpha_{d} \in(-1, \infty) \cap(-m-$ $\varsigma, m+\varsigma)$ [ $]$. 


\section{Preliminaries and Notation}

To smoothen the exposition of the material in main Sections 3 and 4 we gather below some necessary prerequisites and notation.

Let $\mathbb{T}_{s}:=\{z:|z|=s\}, \mathbb{T}_{s}^{ \pm}:=\mathbb{T}_{s} \cap\{z: \pm \operatorname{Im}(z) \geq 0\}$, and $\mathbb{D}_{s}:=\{z:|z|<s\}$, $s>0$, be the circle, the semicircles, and the open disk centered at the origin of radius $s$. For simplicity, we drop the lower index 1 for the unit circle (semicircles) and the unit disk.

Denote by $H^{p}, p \in[1, \infty]$, the Hardy spaces of the unit disk consisting of holomorphic functions $f$ in $\mathbb{D}$ such that

$$
\begin{array}{lll}
\|f\|_{p}^{p}:=\sup _{0<s<1} \frac{1}{2 \pi} \int_{\mathbb{T}}|f(s \xi)|^{p}|d \xi|<\infty & \text { if } & p \in[1, \infty), \\
\|f\|_{\infty}:=\sup _{z \in \mathbb{D}}|f(z)|<\infty & \text { if } & p=\infty .
\end{array}
$$

It is known [12, Thm. I.5.3] that a function in $H^{p}$ is uniquely determined by its trace (non-tangential limit) on the unit circle and that the $L^{p}$-norm of this trace is equal to the $H^{p}$-norm of the function, where $L^{p}$ is the space of $p$-summable functions on $\mathbb{T}$. This way $H^{p}$ can be regarded as a closed subspace of $L^{p}$.

In the same vein, we define $\bar{H}_{0}^{p}, p \in[1, \infty]$, consisting of holomorphic functions in $\overline{\mathbb{C}} \backslash \overline{\mathbb{D}}$ that vanish at infinity and satisfy 2.1 with $1<s<\infty$ and $z \in \overline{\mathbb{C}} \backslash \overline{\mathbb{D}}$, respectively. In particular, we have that $L^{2}=H^{2} \oplus \bar{H}_{0}^{2}$. Thus, we may define orthogonal projections $\mathcal{P}_{+}: L^{2} \rightarrow H^{2}$ (analytic) and $\mathcal{P}_{-}: L^{2} \rightarrow \bar{H}_{0}^{2}$ (antianalytic). It is easy to see that

$$
\int_{\mathbb{T}} \frac{h(\xi)}{\xi-z} \frac{d \xi}{2 \pi i}=\left\{\begin{array}{ll}
\mathcal{P}_{+}(h)(z), & z \in \mathbb{D}, \\
-\mathcal{P}_{-}(h)(z), & z \in \overline{\mathbb{C}} \backslash \overline{\mathbb{D}},
\end{array} \quad h \in L^{2} .\right.
$$

Recall also the well-known fact [12, Cor. II.5.8] that any nonzero function in $H^{p}$ can be uniquely factored as $h=j w$, where

$$
w(z)=\exp \left\{\frac{1}{2 \pi} \int \frac{\xi+z}{\xi-z} \log |h(\xi)||d \xi|\right\}, \quad z \in \mathbb{D},
$$

belongs to $H^{p}$ and is called the outer factor of $h$, while $j$ has modulus 1 a.e. on $\mathbb{T}$ and is called the inner factor of $h$. The latter may be further decompose as $j=b s$, where $b$ is a Blaschke product, i.e. a function of the form

$$
b(z)=z^{k} \prod_{z_{j} \neq 0} \frac{-\bar{z}_{j}}{\left|z_{j}\right|} \frac{z-z_{j}}{1-\bar{z}_{j} z}
$$

that has the same zeroing as $h$, while $s$ is the singular inner factor. For simplicity, we often say that a function is outer (resp. inner) if it is equal to its outer (resp. inner) factor.

Continuing with the notation, for any point-set $K$ and any function $f \in H^{p}$, we denote by $K^{*}$ and $f^{\sigma}$ their reflections across $\mathbb{T}$, i.e., $K^{*}:=\{z: 1 / \bar{z} \in K\}$ and $f^{\sigma}(z):=z^{-1} \overline{f(1 / \bar{z})}$. Clearly then $f \in \bar{H}_{0}^{p}$ and the map ${ }^{\sigma}$ is idempotent. Further, 
for an interval $[c, d]$ we set

$$
\kappa:=4 /(d-c), \quad \mathfrak{w}(z):=\sqrt{(z-c)(z-d)}, \quad \text { and } \quad \mathfrak{D}:=\overline{\mathbb{C}} \backslash[c, d],
$$

where such a branch of $\mathfrak{w}$ is chosen that $\mathfrak{w}$ is holomorphic in $\mathfrak{D} \backslash\{\infty\}$ and $\mathfrak{w}(z) / z \rightarrow$ 1 as $z \rightarrow \infty$. Then

$$
\widetilde{\mathfrak{w}}(z):=1 /(1 / \mathfrak{w}(z))^{\sigma}=z^{2} \mathfrak{w}^{\sigma}(z)=\sqrt{(1-c z)(1-d z)}
$$

is holomorphic in $\mathfrak{D}^{*} \backslash\{\infty\}$ and $\widetilde{\mathfrak{w}}(0)>0$. Moreover, the function

$$
\psi(z):=\frac{2 z-(d+c)-2 \mathfrak{w}(z)}{d-c}, \quad z \in \mathfrak{D},
$$

is the conformal map of $\mathfrak{D}$ onto $\mathbb{D}$ such that $\psi(\infty)=0$ and $\psi^{\prime}(\infty)=\kappa>0$. It is also easy to see that $\psi$ has well-defined unrestricted boundary values from both side of $[c, d]$ (we assume that the positive side of $[c, d]$ lies on the left when the interval is traversed in the positive direction, i.e. from $c$ to $d$ ). Moreover, it holds that

$$
\psi^{+} \psi^{-}=1 \quad \text { on } \quad[c, d]
$$

Let now $h$ be a Dini-continuous non-vanishing complex-valued function on $[c, d]$. Recall that Dini-continuity means

$$
\int_{[0, d-c]} \delta^{-1} \max _{\left|t_{1}-t_{2}\right| \leq \delta}\left|h\left(t_{1}\right)-h\left(t_{2}\right)\right| d \delta<\infty .
$$

It can be easily checked (cf. [7, Sec. 3.3]) that the geometric mean of $h$, i.e.

$$
G_{h}:=\exp \left\{\int \log h(t) d \omega_{[c, d]}(t)\right\},
$$

is independent of the actual choice of the branch of the logarithm and is non-zero. Moreover, the Szegö function of $h$, i.e.

$$
S_{h}(z):=\exp \left\{\frac{\mathfrak{w}(z)}{2} \int \frac{\log h(t)}{z-t} d \omega_{[c, d]}(t)-\frac{1}{2} \int \log h(t) d \omega_{[c, d]}(t)\right\},
$$

$z \in \mathfrak{D}$, also does not depend on the choice of the branch (as long as the same branch is taken in both integrals) and is a non-vanishing holomorphic function in $\mathfrak{D}$ that has continuous boundary values from each side of $[c, d]$ and satisfies

$$
h=G_{h} S_{h}^{+} S_{h}^{-} \text {on }[c, d] \text { and } S_{h}(\infty)=1 .
$$

The continuity of the traces of $S_{h}$ is ensured by the Dini-continuity of $h$, essentially because Dini-continuous functions have continuous conjugates [12, Thm. III.1.3]. In fact, more can be said. Let $a$ be a non-vanishing holomorphic function in $\mathfrak{D}$ that has continuous traces on each side of $[c, d]$ and $a(\infty)=1$. Suppose also that $a^{+} a^{-}=c^{2}$ for some constant $c$. Then the functions $a_{i} \circ \psi:=c / a$ and $a_{e} \circ(1 / \psi):=$ $a / c$ are holomorphic in $\mathbb{D}$ and $\overline{\mathbb{C}} \backslash \overline{\mathbb{D}}$, respectively, have continuous traces on $\mathbb{T}$, $a_{i}(0)=c$ and $a_{e}(\infty)=1 / c$. Moreover, it can be readily verified that the traces of $a_{i}$ and $a_{e}$ coincide. Thus, $a_{i}$ and $a_{e}$ are analytic continuations of each other, from which we deduce by Liouville's theorem that $c=1$ and $a_{i} \equiv a_{e} \equiv 1$. This simple observation implies the following. Let $h$ be a Dini-continuous function on $[c, d]$ 
and $G$ be some constant. If $S$ is a non-vanishing holomorphic function in $\mathfrak{D}$ that assumes value 1 at inifnity, has continuous traces, and is such that $G S^{-} S^{+}=h$ then necessarily $G=G_{h}$ and $S=S_{h}$. It is also true that 2.6 is well-defined whenever $h$ is a non-negative integrable function with integrable logarithm; like, for example, $\hbar$ and $\hbar_{\mathbf{x}}$ defined after 1.2 .

We also emphasize that the Szegő function of a polynomial can be computed in a rather explicit manner as we will now see. Let $v$ be a polynomial with zeros in $\mathfrak{D}, \operatorname{deg}(v) \leq k$. Set

$$
r_{k}(v ; z):=(\psi(z))^{k-\operatorname{deg}(v)} \prod_{e \in \mathbf{z}(v)}\left(\frac{\psi(z)-\psi(e)}{1-\psi(z) \psi(e)}\right)^{m(e)}, \quad z \in \mathfrak{D},
$$

where $\mathrm{z}(v)$ is the set of zeros of $v$ and $m(e)$ is the multiplicity of $e \in \mathrm{z}(v)$. Then $r_{k}(v ; \cdot)$ is a holomorphic function in $\mathfrak{D}$ with a zero of multiplicity $m(e)$ at each $e \in \mathrm{z}(v)$ and a (possible) zero of multiplicity $k-\operatorname{deg}(v)$ at infinity. Moreover, it has unrestricted continuous boundary values from both sides of $[c, d]$ such that

$$
r_{k}^{+}(v ; \cdot) r_{k}^{-}(v ; \cdot)=1
$$

by (2.4). Then since $S_{v}$ is the unique function of Szegö type such that $S_{v}^{+} S_{v}^{-}$is equal to a constant multiple of $v$, it holds that

$$
S_{v}^{2}=\frac{1}{G_{v}} \frac{v \psi^{k}}{r_{k}(v ; \cdot)}
$$

In some cases it will be important to consider the ratio of the boundary values of Szegö functions rather then their product. Hence, we introduce

$$
Q_{h}^{ \pm}(t):=S_{h}^{ \pm}(t) / S_{h}^{\mp}(t), \quad t \in[c, d] .
$$

When $h$ is non-vanishing Dini-continuous function, $Q_{h}^{ \pm}$are continuous on $[c, d]$ and assume the value 1 at the endpoints. Finalizing the discussion on Szegö functions, let us state two of their properties that we shall use implicitly on several occasions and the reader will have no difficulty to verify. The first one is the multiplicativity property, i.e. $S_{h_{1} h_{2}}=S_{h_{1}} S_{h_{2}}$, and the second one is the convergence property which says that $S_{h_{n}}=[1+o(1)] S_{h}$ uniformly in $\overline{\mathbb{C}}$, i.e. including the boundary values, whenever $h_{n}=[1+o(1)] h$ uniformly on $[c, d]$. For more information on Szegö functions of complex $h$, the reader may consult [7, Sec. 3.3].

Next, we denote by $\mathbb{A}_{s_{1}, s_{2}}:=\left\{z: s_{1}<|z|<s_{2}\right\}, 0<s_{1}<s_{2}$, and $\mathbb{A}_{s}:=\mathbb{A}_{s, 1 / s}, s<1$, the annuli centered at the origin and by $\varphi$ the conformal map from $\mathfrak{D} \cap \mathfrak{D}^{*}$ onto $\mathbb{A}_{\rho}, \varphi(1)=1$. Recall that annuli $\mathbb{A}_{s}$ are not conformally equivalent for different $s$ and therefore $\rho=\rho([c, d])$ is uniquely determined by $[c, d]$. From the potential-theoretic point of view $\rho$ can be expressed as

$$
\rho=\exp \left\{-\frac{1}{\operatorname{cap}([c, d], \mathbb{T})}\right\}
$$


where $\operatorname{cap}([a, b], \mathbb{T})$ is the capacity of the condenser $([c, d], \mathbb{T})$. The map $\varphi$ is given by [24, Thm. VIII.6.1]

$$
\varphi(z)=\exp \left\{\mathcal{T}^{2} \int_{1}^{z} \frac{d t}{(\mathfrak{w} \widetilde{\mathfrak{w}})(t)}\right\}
$$

with integration taken along any path in $\mathfrak{D} \cap \mathfrak{D}^{*}$, where

$$
\mathcal{T}^{-2}:=\frac{2}{\pi} \int_{[0,1]} \frac{d x}{\sqrt{\left(1-x^{2}\right)\left((1-c d)^{2}-(d-c)^{2} x^{2}\right)}} .
$$

Moreover, it holds that $\varphi(\bar{z})=\overline{\varphi(z)}$ and $\varphi(1 / z)=1 / \varphi(z), z \in \mathfrak{D} \cap \mathfrak{D}^{*}$. Thus, $\varphi(\mathbb{T})=\mathbb{T}$ and $\varphi(\mathbb{D} \backslash[c, d])=\mathbb{A}_{\rho, 1}$. Further, it is not hard to check that $\varphi$ extends continuously on each side of $[c, d]$ (resp. $\left.[c, d]^{*}\right)$ and $\varphi^{ \pm}([c, d])=\mathbb{T}_{\rho}^{ \pm}$(resp. $\left.\varphi^{ \pm}\left([c, d]^{*}\right)=\mathbb{T}_{1 / \rho}^{ \pm}\right)$. Finally, the Green equilibrium distribution (which is a probability measure on $[c, d][24$, Sec. II.5]) for the condenser $\mathbb{D} \backslash[c, d]$, as well as for the condenser $\mathfrak{D} \cap \mathfrak{D}^{*}$, is given by

$$
d \omega_{([c, d], \mathbb{T})}(t)=\frac{\mathcal{T}^{2} d t}{\pi\left|\left(\mathfrak{w}^{+} \widetilde{\mathfrak{w}}\right)(t)\right|}=\frac{\left|d \varphi^{+}(t)\right|}{\pi \rho},
$$

where the normalization follows from (2.14) and the second equality holds by differentiating (2.13) and taking boundary values.

\section{Meromorphic Approximation}

The meromorphic approximants (MAs) that we deal with are defined as follows. For $p \in[1, \infty]$ and $n \in \mathbb{N}$, the class of meromorphic functions of degree $n$ in $L^{p}$ is

$$
H_{n}^{p}:=H^{p} B_{n}^{-1},
$$

where $B_{n}$ is the set of Blaschke products of degree at most $n$ (with at most $n$ zeros). By the celebrated theorem of Adamyan, Arov, and Krein [1] (see also 20, Ch. 4]) and its generalizations [5, 21] it is known that for any fixed $n \in \mathbb{N}$ and $p \in[1, \infty]$ and given $f \in L^{p}$ there exists a meromorphic function $g_{n}$ such that

$$
\left\|f-g_{n}\right\|_{p}=\inf _{g \in H_{n}^{p}}\|f-g\|_{p} .
$$

Moreover, $g_{n}$ is unique when $p \in[1, \infty)$, but in the case $p=\infty$ it is necessary to assume $f \in H^{\infty}+C(\mathbb{T})$ to ensure uniqueness of $g_{n}$, where $C(\mathbb{T})$ is the space of continuous functions on the unit circle. Obviously, when $\operatorname{supp}(\mu) \subset \mathbb{D}$ and $q$ has no zeros on $\mathbb{T}$ the function $\mathfrak{f}$ defined in (1.1) complies with these requirements for any $p \in[1, \infty]$. When $p<2$, no functional representation for the error is known to satisfy orthogonality relations [5]. This is the reason why in what follows we shall restrict to the case $p \in[2, \infty]$. 
Due to similar functional decomposition and their appearances in the computations ${ }^{1}$, we consider not only best MAs but more generally critical point of meromorphic approximation problem 3.2. Although their definition is rather technical (see below), critical points are just those $g_{n}=h_{n} / b_{n} \in H_{n}^{p}$ (see (3.1) for which the derivative of $\left\|f-g_{n}\right\|_{p}$ with respect to $b_{n} \in B_{n}$ and $h_{n} \in H^{p}$ does vanish 5. By definition, a function $g_{n}$ is a critical point of order $n$ in meromorphic approximation problem 3.2 if and only if it assumes the form

$$
g_{n}=f-\frac{\mathcal{H}_{f}\left(v_{n}\right)}{v_{n}}=\frac{\mathcal{P}_{+}\left(f v_{n}\right)}{v_{n}},
$$

where $\mathcal{H}_{f}$ is Hankel operator with a symbol $f$, i.e.

$$
\mathcal{H}_{f}: H^{p^{\prime}} \rightarrow \bar{H}_{0}^{2}, \quad \mathcal{H}_{f}(h):=\mathcal{P}_{-}(f h), \quad 1 / p+1 / p^{\prime}=1 / 2,
$$

and $v_{n} \in H^{p^{\prime}}$ is of unit norm (a Blaschke product if $p=2$ ), its inner factor is a Blaschke product of exact degree $n$, and is such that

$$
\begin{array}{ll}
\mathcal{H}_{f}^{*} \mathcal{H}_{f}\left(v_{n}\right)=\sigma_{n}^{2} \mathcal{P}_{+}\left(\left|v_{n}\right|^{p^{\prime}-2} v_{n}\right) & \text { if } p>2, \\
\mathcal{H}_{f}^{*} \mathcal{H}_{f}\left(v_{n}\right)=\mathcal{P}_{+}\left(\left|\mathcal{H}_{f}\left(v_{n}\right)\right|^{2} v_{n}\right) & \text { if } p=2,
\end{array}
$$

with $\mathcal{H}_{f}^{*}$ being the adjoint operator. A function $v_{n}$ is called a singular vector associated to a critical point $g_{n}$ and

$$
\sigma_{n}:=\left\|f-g_{n}\right\|_{p}=\left\|\mathcal{H}_{f}\left(v_{n}\right)\right\|_{p}, \quad p \in[2, \infty],
$$

is called the critical value associated to $g_{n}$. In the case when $g_{n}$ is a best MA to $f$ it also holds that $\sigma_{n}$ is the $n$-th singular number of $\mathcal{H}_{f}$, i.e.

$$
\sigma_{n}=\sigma_{n}\left(\mathcal{H}_{f}\right):=\inf \left\{\left\|\mathcal{H}_{f}-\Gamma\right\|: \Gamma: H^{p^{\prime}} \rightarrow \bar{H}_{0}^{2} \text { linear operator of rank } \leq n\right\} ;
$$

when $p=2$ it is assumed in addition that $\Gamma$ is weak* continuous. Hereafter, we use the following notation for the inner-outer decomposition of singular vectors:

$$
v_{n}=b_{n} w_{n}, \quad b_{n}=q_{n} / \widetilde{q}_{n}, \quad w_{n}(0)>0, \quad \widetilde{q}_{n}(z)=z^{n} \overline{q_{n}(1 / \bar{z})}
$$

where $w_{n}$ is an outer factor, $q_{n}$ is a monic polynomial of exact degree $n$, and $\widetilde{q}_{n}$ is the reciprocal polynomial of $q_{n}$. To uniformize the notation, we simply set $w_{n} \equiv 1$ when $p=2$.

A critical point of order $n$ may have less than $n$ poles, even though we insisted in the definition that $v_{n}$ has exactly $n$ zeros. Cancellation may occur due to zeros of $\mathcal{P}_{+}\left(f v_{n}\right)$. When this is not the case, we shall call $g_{n}$ an irreducible critical point. It is worth mentioning that when $p \in[2, \infty)$ a best MA is not necessarily unique, but has exactly $n$ poles. Thus, all best MAs are irreducible critical points. To the contrary, if $p=\infty$, best MA is unique and is the only critical point of order $n$, but may have less then $n$ poles. However, there always exists a subsequence of natural numbers for which best AAK approximants are irreducible. Since the behavior of the poles of MAs is entirely characterized by this subsequence, hereafter we say

${ }^{1}$ It is most likely that a numerical search ends up with stable critical points, that is locally best MAs, rather than just best MAs. 
"a sequence of irreducible critical points" to mean if $p=\infty$ that we pass to a subsequence if needed.

Now, we are ready to state the first theorem of this section.

Theorem 1. Let $\mathfrak{f}$ be given by (1.1) and 1.2a). Further, let $\left\{g_{n}\right\}$ be a sequence of irreducible critical points of the meromorphic approximation problem to $\mathfrak{f}, p \in$ $(2, \infty]$. Then the outer factors $w_{n}$ in 3.5 are such that

$$
w_{n}^{p^{\prime} / 2}=\frac{\mathcal{T}+o(1)}{\widetilde{\mathfrak{w}}}+\frac{l_{n}}{\widetilde{q}}, \quad \widetilde{q}(z)=z^{m} \overline{q(1 / \bar{z})},
$$

where o(1) holds locally uniformly in $\mathfrak{D}^{*}, \mathcal{T}$ was defined in (2.14, and the polynomials $l_{n}, \operatorname{deg}\left(l_{n}\right)<m$, converge to zero and are coprime with $\widetilde{q}$.

This theorem is a strengthening of Lemma 3.4 in [9] that asserts, under much milder assumptions on $\mu$, that $\left\{w_{n}^{p^{\prime} / 2}\right\}$ is a normal family in $\mathfrak{D}_{\mathfrak{f}}^{*}$ and any limit point of $\left\{w_{n}\right\}$ in $\mathbb{D}$ is zero free.

For simplicity, set $w:=(\mathcal{T} / \widetilde{\mathfrak{w}})^{2 / p^{\prime}}$ for each $p>2$. Then Theorem 11 yields that $w_{n} \rightarrow w$, uniformly in some neighborhood of $\overline{\mathbb{D}}$ (it follows from the proof of Theorem 1 and can be seen from asymptotic formula 3.6 that the outer factors $w_{n}$ can be extended to holomorphic functions in any simply connected neighborhood of $\overline{\mathbb{D}}$ contained in $\mathfrak{D}_{\mathfrak{f}}^{*}$ ). Now, we are ready to describe the asymptotic behavior of irreducible critical points.

Theorem 2. Let $\mathfrak{f}$ and $\left\{g_{n}\right\}$ be as in Theorem 1. Then the Blaschke products $b_{n}$ in (3.5) are such that

$$
b_{n}=[1+o(1)] b \varphi^{n-m} D_{n}^{-1} \quad \text { locally uniformly in } \quad \mathfrak{D}_{\mathfrak{f}} \cap \mathfrak{D}_{\mathfrak{f}}^{*},
$$

where $b:=q / \widetilde{q}, m=\operatorname{deg}(q),\left\{D_{n}\right\}$ is a normal family of non-vanishing functions in $\mathfrak{D} \cap \mathfrak{D}^{*}$, such that $\left|D_{n}^{ \pm}\right|$are uniformly bounded above and away from zero on $[c, d]$ and $[c, d]^{*}$. Moreover, the following error estimates take place

$$
\sigma_{n}=\left\|\mathfrak{f}-g_{n}\right\|_{p}=\left[2 M \mathcal{T}^{-1}+o(1)\right] \rho^{2(n-m)},
$$

where $\sigma_{n}$ is the critical value associated to $g_{n}$ via (3.4), and

$$
\left(\mathfrak{f}-g_{n}\right)=\frac{2 M+o(1)}{w \mathfrak{w}}\left(\frac{\rho}{\varphi}\right)^{2(n-m)} \frac{D_{n}^{2}}{b^{2}}
$$

uniformly on compact subsets of $\mathfrak{D}_{\mathfrak{f}} \cap \overline{\mathbb{D}}$, where

$$
M:=\exp \left\{\int \log \left|\left(b^{2} w \dot{\mu}\right)(t)\right| d \omega_{([c, d], \mathbb{T})}(t)\right\},
$$

is the geometric mean of $\left|b^{2} w \dot{\mu}\right|$ with respect to the condenser $\mathfrak{D} \cap \mathfrak{D}^{*}$.

It is worth mentioning that the functions $D_{n}$ are, in fact, Szegö functions for the condenser $\mathfrak{D} \cap \mathfrak{D}^{*}$ that first were introduced in [6, Def. 2.38] for the case of a positive measure $\mu$. In such a situation the Szegö function for a condenser 
has an integral representation that is no longer valid for complex measures. Moreover, the normalization in the complex case is more intricate (see Proposition 11). Nevertheless, it still holds that the functions $D_{n}$ have zero winding number on any curve separating $[c, d]$ from $[c, d]^{*}, D_{n}(z) \overline{D_{n}(1 / \bar{z})}=1, z \in \mathfrak{D} \cap \mathfrak{D}^{*}$, and $M\left|D_{n}^{+} D_{n}^{-}\right|=\left|b^{2} w \dot{\mu}\right|$ on $[c, d]$.

We remind the reader that the case $p=2$ has a couple of special traits. First, best MA $g_{n}$ specializes to a rational function. Indeed, $g_{n}$ can be written as a sum $h_{n}+p_{n-1} / q_{n}$, where $h_{n} \in H^{2}, \operatorname{deg}\left(p_{n-1}\right)<\operatorname{deg}\left(q_{n}\right)=n$. As $L^{2}=H^{2} \oplus \bar{H}_{0}^{2}$ and $\mathfrak{f} \in \bar{H}_{0}^{2}$, we have that

$$
\|\mathfrak{f}-g\|_{2}^{2}=\|h\|_{2}^{2}+\left\|\mathfrak{f}-p_{n-1} / q_{n}\right\|_{2}^{2} .
$$

Hence, to achieve the minimum of the left-hand side of the equality above, one necessarily should take $h \equiv 0$. This is the reason why we referred on some occasions to the meromorphic approximation problem with $p=2$ as to the rational approximation problem. Second, the outer factors $w_{n}$ in 3.5 are not present, or better assumed to be identically 1 . The latter allows us to consider a slightly larger class of measures, namely those given by $(1.2 \mathrm{~b})$.

Theorem 3. Let $\mathfrak{f}$ be given by (1.1) and $1.2 \mathrm{~b}$, where $\sin \left(\alpha_{x} \pi\right) \in\left(0, \Psi_{x}\right)$,

$$
\Psi_{x}:=\min _{ \pm}\left\{\left|Q_{q^{2} h}^{ \pm}(x)\right|\right\} \exp \left\{-\frac{4 s_{1}\left[V_{h}+2 m \pi\right]}{1-s_{0}}\right\}, \quad x \in \mathbf{x},
$$

$V_{h}$ is the total variation of the argument of $h$ on $[c, d], s_{0}:=\max _{\mathbb{T}}|\psi|$, and $s_{1}:=\max _{\mathbb{T}}\left|\psi^{\prime}\right|$. Let further $\left\{g_{n}\right\}$ be a sequence of irreducible critical points of the meromorphic approximation problem with $p=2$ to $\mathfrak{f}$. Then (3.6), (3.7), and (3.8) hold with $w \equiv 1$.

It follows from (3.7) that each $b_{n}$ has exactly $m$ zeros approaching the zeros of $b$. In fact, it is possible to say more.

Theorem 4. For each $\eta \in \mathrm{z}(q)$ and all $n$ large enough, there exists an arrangement of $\eta_{1, n}, \ldots \eta_{m(\eta), n}$, the zeros of $b_{n}$ approaching $\eta$, such that

$$
\eta_{k, n}=\eta+A_{k, n}^{\eta}\left(\frac{\rho}{\varphi(\eta)}\right)^{2(n-m) / m(\eta)} \exp \left\{\frac{2 \pi k i}{m(\eta)}\right\}, \quad k=1, \ldots, m(\eta),
$$

where the sequences $\left\{\max _{k}\left|A_{k, n}^{\eta}\right|\right\}$ and $\left\{\max _{k}\left|1 / A_{k, n}^{\eta}\right|\right\}$ are bounded above.

This theorem essentially says that each pole $\eta$ of $\mathfrak{f}$ attracts exactly $m(\eta)$ poles of $g_{n}$, the latter converge geometrically fast and are asymptotically distributed as the roots of unity of order $m(\eta)$. The proof of this theorem is an adaptation of the technique developed in 14 for classical Padé approximants to Cauchy transforms of analytic densities. As one can see from the next section, similar results hold not only for classical but more generally for multipoint Padé approximants to Cauchy transforms of less regular measures. 


\section{Multipoint Padé Approximation}

Let $\mathfrak{f}$ be given by 1.1 . Classically, diagonal (multipoint) Padé approximants to $\mathfrak{f}$ are rational functions of type $(n, n)$ that interpolate $\mathfrak{f}$ at a prescribed system of $2 n+1$ points. However, when the approximated function is of the form 1.1 , it is customary to place at least one interpolation condition at infinity. More precisely, let $\mathscr{E}=\left\{E_{n}\right\}$ be a sequence of sets each consisting of $2 n$ not necessarily distinct nor finite points in $\mathfrak{D}_{\mathfrak{f}}$ (interpolation scheme), and let $v_{n}$ be the monic polynomial with zeros at the finite points of $E_{n}$.

Definition (Padé Approximants). Given $\mathfrak{f}$ of type (1.1) and an interpolation scheme $\mathscr{E}$, the $n$-th diagonal Padé approximant to $\mathfrak{f}$ associated with $\mathscr{E}$ is the unique rational function $\Pi_{n}=p_{n} / q_{n}$ satisfying:

- $\operatorname{deg} p_{n} \leq n, \operatorname{deg} q_{n} \leq n$, and $q_{n} \not \equiv 0$;

- $\left(q_{n}(z) \mathfrak{f}(z)-p_{n}(z)\right) / v_{n}(z)$ is analytic in $\mathfrak{D}_{\mathfrak{f}}$;

- $\left(q_{n}(z) \mathfrak{f}(z)-p_{n}(z)\right) / v_{n}(z)=O\left(1 / z^{n+1}\right)$ as $z \rightarrow \infty$.

A Padé approximant always exists since the conditions for $p_{n}$ and $q_{n}$ amount to solving a system of $2 n+1$ homogeneous linear equations with $2 n+2$ unknown coefficients, no solution of which can be such that $q_{n} \equiv 0$ (we may thus assume that $q_{n}$ is monic); note the required interpolation at infinity is entailed by the last condition and therefore $\Pi_{n}$ is, in fact, of type $(n-1, n)$.

By the very definition, the behavior of $\Pi_{n}$ depends on the choice of the interpolation scheme. We define the support of $\mathscr{E}=\left\{E_{n}\right\}$ as $\operatorname{supp}(\mathscr{E}):=\cap_{n \in \mathbb{N}} \overline{\bigcup_{k \geq n} E_{k}}$. Hereafter, the counting measure of a finite set is a probability measure that has equal mass at each point counting multiplicities and the weak* topology is understood with respect to the duality between complex measures and continuous functions with compact support in $\overline{\mathbb{C}}$.

Definition (Admissibility). An interpolation scheme $\mathscr{E}$ is called admissible if

- there exist rearrangements $\Delta_{n}$ of $E_{n}$ such that the sums $\sum_{e \in E_{n}} \mid \psi(\bar{e})-$ $\psi\left(\Delta_{n}(e)\right) \mid$ are uniformly bounded when $n \rightarrow \infty$;

- $\operatorname{supp}(\mathscr{E}) \subset \mathfrak{D}_{\mathfrak{f}}$ and the probability counting measures of points in $E_{n}$ converge weak* $^{*}$ to some Borel measure with finite Green energy ${ }^{2}$ relative to $\mathfrak{D}$.

Then the following result holds.

Theorem 5. Let $\left\{\Pi_{n}\right\}$ be a sequence of diagonal Padé approximants associated with an admissible interpolation scheme $\mathscr{E}=\left\{E_{n}\right\}$ to $\mathfrak{f}$ given by 1.1 and $[1.2 \mathrm{~b}$ with

$$
\alpha_{x} \pi \in\left(0, \arcsin \Upsilon_{x}\right), \quad \Upsilon_{x}:=\liminf _{n \rightarrow \infty} \min _{ \pm}\left\{\left|\left(r_{n} Q_{h}\right)^{ \pm}(x)\right|\right\}
$$

for any $x \in \mathbf{x}$, where $r_{n}:=r_{2 n}\left(v_{n} ; \cdot\right)$. Then

$$
\left(\mathfrak{f}-\Pi_{n}\right) \mathfrak{w}=\left[2 G_{\dot{\mu}}+o(1)\right] S_{\dot{\mu}}^{2} \frac{r_{n}}{r^{2}}
$$

\footnotetext{
${ }^{2}$ For information on the notions of potential theory we refer the reader to the monographs 23,24$]$.
} 
locally uniformly in $\mathfrak{D}_{\mathfrak{f}}$, where $r:=r_{m}(q ; \cdot)$. When $(p / q) \equiv 0$ it is not necessary to assume boundedness of the variation of the argument of $h$.

We would like to point out that $\Upsilon_{x}$ is, in fact, continuous function of $x$ on $[c, d]$ such that $\Upsilon_{c}=\Upsilon_{d}=1$ and $\min _{x \in[c, d]} \Upsilon_{x}>0$. The latter is true since the functions $Q_{h}^{ \pm}$are non-vanishing and continuous on $[c, d]$. Moreover, it will be shown in the proof of Theorem 7 that the admissibility of $\mathscr{E}$ implies uniform boundedness of $\left|r_{n}^{ \pm}\right|$and hence their uniform boundedness away from zero by 2.9 ). It is also easy to check that when the sets $E_{n}$ are conjugate-symmetric and $h$ is a positive function, it holds that $\Upsilon_{x} \equiv 1$.

Concerning the behavior of $\Pi_{n}$ near polar singularities of $\mathfrak{f}$, i.e. near $\mathrm{z}(q)$, the following theorem asserts the same "roots of unity" behavior as in Theorem 4 and is a generalization of [14, Thm. 3] for the case of multipoint Padé approximants and less regular measures.

Theorem 6. Under the conditions of Theorem 5 let $q_{n}$ be the denominators of $\Pi_{n}$. Then

$$
q_{n}=u_{n-m} q_{n, m} \quad \text { and } \quad q_{n, m}=(1+o(1)) q,
$$

where $\operatorname{deg}\left(u_{n-m}\right)=n-m, \operatorname{deg}\left(q_{n, m}\right)=m$, the polynomials $u_{n-m}$ have no zeros on any closed set in $\mathfrak{D}$ for all $n$ large enough, and o(1) holds locally uniformly in $\overline{\mathbb{C}} \backslash \mathrm{z}(q)$. Moreover, for each $\eta \in \mathrm{z}(q)$ with multiplicity $m(\eta)$ and all $n$ large enough there exists an arrangement of $\eta_{1, n}, \ldots, \eta_{m(\eta), n} \in \mathrm{z}\left(q_{n, m}\right)$ such that

$$
\eta_{k, n}=\eta+A_{k, n}^{\eta}\left[r_{n}(\eta)\right]^{1 / m(\eta)} \exp \left\{\frac{2 \pi k i}{m(\eta)}\right\}, \quad k=1, \ldots, m(\eta),
$$

where the sequence $\left\{\max _{k}\left|A_{k, n}^{\eta}\right|\right\}$ is bounded above.

\section{Non-Hermitian Orthogonal Polynomials}

In this section we describe the asymptotic behavior of non-Hermitian orthogonal polynomials with varying weights on $[c, d]$. In what follows, we assume that $\left\{\nu_{n}\right\}$ is a sequence of complex measures on $[c, d]$ such that

$$
d \nu_{n}=\dot{\nu}_{n} d \omega_{[c, d]}, \quad \dot{\nu}_{n}=h h_{n} \hbar \hbar_{\mathbf{x}} / v_{n+m}, \quad m \in \mathbb{Z}_{+},
$$

where $h$ is a non-vanishing Dini-continuous function on $[c, d],\left\{h_{n}\right\}$ is a normal family of non-vanishing functions in some neighborhood of $[c, d]$ none of which limit points can vanish in this neighborhood, $v_{n}, \operatorname{deg}\left(v_{n}\right) \leq 2 n$, are monic polynomials with zeros at finite points of an admissible interpolation scheme, $\hbar$ and $\hbar_{\mathbf{x}}$ are as in the introduction with

$$
\alpha_{x} \pi \in\left(0, \arcsin \Upsilon_{x}\right), \quad \Upsilon_{x}:=\liminf _{n \rightarrow \infty} \min _{ \pm}\left\{\left|\left(r_{n+m} Q_{h h_{n}}\right)^{ \pm}(x)\right|\right\},
$$

for each $x \in \mathbf{x}$, where $r_{k}:=r_{2 k}\left(v_{k} ; \cdot\right)$. Observe that we do not require $h$ to have argument of bounded variation. Then the following theorem holds. 
Theorem 7. Let $\left\{\nu_{n}\right\}$ be as described and $\left\{u_{n}\right\}$ be a sequence of polynomials satisfying

$$
\int t^{j} u_{n}(t) d \nu_{n}(t)=0, \quad j=0, \ldots, n-1
$$

and $\left\{R_{n}\right\}$ be the sequence of corresponding functions of the second kind, i.e.,

$$
R_{n}(z):=\int \frac{u_{n}(t)}{z-t} d \nu_{n}(t)=\frac{1}{u_{n}(z)} \int \frac{u_{n}^{2}(t)}{z-t} d \nu_{n}(t)
$$

Then, for all $n$ large enough, the polynomials $u_{n}$ have exact degree $n$ and therefore can be normalized to be monic. Under such a normalization it holds that

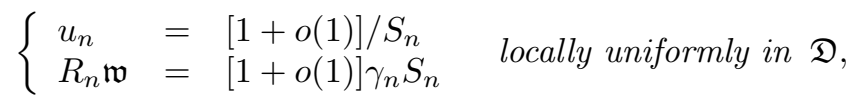

where $S_{n}:=S_{\dot{\nu}_{n}}(\kappa \psi)^{n}, \gamma_{n}:=2 \kappa^{-2 n} G_{\dot{\nu}_{n}}$, and $\kappa$ and $\mathfrak{w}$ were defined in 2.2.

Proof. This theorem is an adaptation of [7, Thm. 3]. To see this we need several observations. Firstly, the orthogonality relations in [7, Thm. 3] are considered on Jordan arcs connecting -1 and 1 , of which the interval $[-1,1]$ is a particular case. The current setting can be easily deduced by applying a linear transformation $l(x)=[(d-c) x+d+c] / 2$.

Secondly, $\left\{h_{n}\right\}$ is taken in [7, Thm. 3] to be a family of Dini-continuous non-vanishing functions on $[c, d]$ such that any sequence in this family contains a uniformly convergent subsequence to a non-vanishing function and the moduli of continuity of $h_{n}$ are bounded by the same fixed modulus of continuity. Clearly, the normality of $\left\{h_{n}\right\}$ yields that all these restrictions are met in the present case.

Thirdly, only the case $m=0$ is considered in [7, Thm. 3]. However, the general case we are dealing with is no different. Indeed, choose $2 m$ zeros of each polynomial $v_{n+m}$ that converge to some fixed point in $\mathfrak{D}$ (recall that the counting measures of zeros of $v_{n}$ converge in the weak* sense) and pay the polynomial, say $p_{2 m, n}$, vanishing at these points to $h_{n}$. Then $\left\{h_{n} / p_{2 m, n}\right\}$ is again, a normal family of holomorphic functions with the required properties and the new polynomial factor $v_{n+m} / p_{2 m}$ of $\dot{\nu}_{n}$ has degree no greater than $2 n$.

Finally, in order to appeal to [7, Thm. 3], we need to show that the functions $r_{n}=r_{2 n}\left(v_{n} ; \cdot\right)$ are such that $r_{n}=o(1)$ locally uniformly in $\mathfrak{D},\left|r_{n}^{ \pm}\right|=O(1)$ on $[c, d]$, and the moduli of continuity of $\left|r_{n}^{ \pm} \circ \psi^{-1}\right|$ are bounded by the same fixed modulus of continuity ${ }^{3}$ To do so, consider

$$
\hat{r}_{n}(z):=r_{n}\left(\psi^{-1}(z)\right)=\prod_{c \in \hat{E}_{n}} \frac{z-c}{1-c z}, \quad \hat{E}_{n}:=\left\{\psi(e): e \in E_{n}\right\}, \quad z \in \mathbb{D} .
$$

Observe that the sets $E_{n}$ lie at fixed positive distance from $[c, d]$ by the assumption $\operatorname{supp}(\mathscr{E}) \subset \mathfrak{D}_{\mathfrak{f}}$ and therefore there exists $s_{1}<1$ such that $\hat{E}_{n} \subset \mathbb{D}_{s_{1}}$ for all $n$. Thus,

${ }^{3}$ Observe that $\psi^{-1}$ is just the Joukovski transformation. 
the Blaschke products

$$
\hat{b}_{n}(z):=\prod_{c \in \hat{E}_{n}} \frac{z-c}{1-\bar{c} z}=o(1)
$$

locally uniformly in $\mathbb{D}[12$, Thm. 2.2.1]. Further,

$$
\left|\psi\left(z_{1}\right)-\psi\left(z_{2}\right)\right| \leq \max _{z \in K}\left|\psi^{\prime}(z)\right|\left|z_{1}-z_{2}\right|, \quad z_{1}, z_{2} \in K:=\psi^{-1}\left(\overline{\mathbb{D}}_{s_{1}}\right),
$$

and we get from the admissibility of $\left\{E_{n}\right\}$ that

$$
\sum_{c \in \hat{E}_{n}}\left|\bar{c}-\hat{\Delta}_{n}(c)\right| \leq s_{2}, \quad \hat{\Delta}_{n}(c):=\psi\left(\Delta_{n}\left(\psi^{-1}(c)\right)\right),
$$

for all $n$ and some positive constant $s_{2}$. Consider now the functions

$$
\left(\hat{r}_{n} / \hat{b}_{n}\right)(z)=\prod_{c \in \hat{E}_{n}} \frac{1-\bar{c} z}{1-c z}=\prod_{c \in \hat{E}_{n}} \frac{1-\bar{c} z}{1-\hat{\Delta}_{n}(c) z}, \quad z \in \overline{\mathbb{D}} .
$$

Clearly, this is a sequence of outer functions in $\mathbb{D}$. Moreover,

$$
\log \left|\left(\hat{r}_{n} / \hat{b}_{n}\right)(\tau)\right|=\sum_{c \in \hat{E}_{n}} \log \left|1+\frac{\left(\hat{\Delta}_{n}(c)-\bar{c}\right) \tau}{1-\hat{\Delta}_{n}(c) \tau}\right| \leq \sum_{c \in \hat{E}_{n}} \frac{\left|\hat{\Delta}_{n}(c)-\bar{c}\right|}{1-\left|\hat{\Delta}_{n}(c)\right|} \leq \frac{s_{2}}{1-s_{1}}
$$

for $\tau \in \mathbb{T}$. Thus, we have that

$$
\left|\hat{r}_{n}\right| \leq s_{3}\left|\hat{b}_{n}\right|=o(1), \quad s_{3}:=\exp \left\{s_{2} /\left(1-s_{1}\right)\right\},
$$

locally uniformly in $\mathbb{D}$ and

$$
\left|\hat{r}_{n}\right|=\left|\hat{r}_{n} / \hat{b}_{n}\right| \leq s_{3} \quad \text { on } \quad \mathbb{T} .
$$

Therefore, the corresponding properties of $r_{n}$ and $\left|r_{n}^{ \pm}\right|$follow.

Next, we show that $\left|r_{n}^{ \pm} \circ \psi^{-1}\right|$ have moduli of continuity majorized by the same function. As $\left|\hat{b}_{n}\right| \equiv 1$ on $\mathbb{T}$, it is enough to consider $\left|\hat{r}_{n} / \hat{b}_{n}\right|$. Let $\tau_{1}, \tau_{2} \in \mathbb{T}$. Then

$$
\log \left|\frac{\left(\hat{r}_{n} / \hat{b}_{n}\right)\left(\tau_{2}\right)}{\left(\hat{r}_{n} / \hat{b}_{n}\right)\left(\tau_{1}\right)}\right|=\sum_{c \in \hat{E}_{n}} \log \left|1+\frac{\left(\tau_{1}-\tau_{2}\right)\left(\bar{c}-\hat{\Delta}_{n}(c)\right)}{\left(1-\bar{c} \tau_{1}\right)\left(1-\hat{\Delta}_{n}(c) \tau_{2}\right)}\right| \leq \frac{s_{2}\left|\tau_{1}-\tau_{2}\right|}{\left(1-s_{1}\right)^{2}} .
$$

Therefore, we have with $s_{4}:=s_{2} /\left(1-s_{1}\right)^{2}$ that

$$
\exp \left\{-s_{4}\left|\tau_{1}-\tau_{2}\right|\right\} \leq\left|\frac{\left(\hat{r}_{n} / \hat{b}_{n}\right)\left(\bar{\tau}_{1}\right)}{\left(\hat{r}_{n} / \hat{b}_{n}\right)\left(\bar{\tau}_{2}\right)}\right|=\left|\frac{\left(\hat{r}_{n} / \hat{b}_{n}\right)\left(\tau_{2}\right)}{\left(\hat{r}_{n} / \hat{b}_{n}\right)\left(\tau_{1}\right)}\right| \leq \exp \left\{s_{4}\left|\tau_{1}-\tau_{2}\right|\right\} .
$$

Moreover, denoting by $\operatorname{Arg}(z) \in(-\pi, \pi]$ the principal argument of $z \neq 0$ and using

$$
|\operatorname{Arg}(1+z)| \leq \arcsin |z| \leq \pi|z| / 2, \quad|z|<1,
$$

we get that

$$
\left|\operatorname{Arg}\left(\frac{\left(\hat{r}_{n} / \hat{b}_{n}\right)\left(\tau_{2}\right)}{\left(\hat{r}_{n} / \hat{b}_{n}\right)\left(\tau_{1}\right)}\right)\right| \leq \sum_{c \in \hat{E}_{n}}\left|\operatorname{Arg}\left(1+\frac{\left(\tau_{1}-\tau_{2}\right)\left(\bar{c}-\hat{\Delta}_{n}(c)\right)}{\left(1-\bar{c} \tau_{1}\right)\left(1-\hat{\Delta}_{n}(c) \tau_{2}\right)}\right)\right| \leq \frac{s_{4}\left|\tau_{1}-\tau_{2}\right|}{2 / \pi}
$$


for $s_{4}\left|\tau_{1}-\tau_{2}\right| \leq 1$. Hence, for such $\tau_{1}$ and $\tau_{2}$ we obtain that

$$
\left|\left(\hat{r}_{n} / \hat{b}_{n}\right)\left(\tau_{1}\right)-\left(\hat{r}_{n} / \hat{b}_{n}\right)\left(\tau_{2}\right)\right| \leq s_{3}\left|1-\frac{\left(\hat{r}_{n} / \hat{b}_{n}\right)\left(\tau_{2}\right)}{\left(\hat{r}_{n} / \hat{b}_{n}\right)\left(\tau_{1}\right)}\right| \leq s_{5}\left|\tau_{1}-\tau_{2}\right|
$$

for some absolute constant $s_{5}$. This finishes the proof of the theorem, granted 7 , Thm. 3].

\section{Proofs of Theorems $1-4$}

We start by providing several auxiliary results.

Lemma 8. Under the conditions of either Theorem 2 or Theorem 3 it holds that

$$
q_{n}=u_{n-m} q_{n, m}, \quad q_{n, m}=(1+o(1)) q,
$$

locally uniformly in $\overline{\mathbb{C}} \backslash \mathrm{z}(q)$, where $\operatorname{deg}\left(u_{n-m}\right)=n-m$ and $\operatorname{deg}\left(q_{n, m}\right)=m$. Moreover, the zeros of the polynomials $\widetilde{q}_{n}^{2}$ form an admissible interpolation scheme.

Proof. It follows from [9, Thm. 2.4] that if $\operatorname{supp}(\mu)$ is a regular set with respect to the Dirichlet problem, $\dot{\mu}$ has an argument of bounded variation on $\operatorname{supp}(\mu)$, and $|\mu|([x-\delta, x+\delta]) \geq l \delta^{L}$ for all $x \in[c, d]$ and some fixed positive constants $l$ and $L$, then in any neighborhood of $\eta \in \mathrm{z}(q)$ the polynomials $q_{n}$ have at least $m(\eta)$ zeros for all $n$ large enough (in fact, no more then $m(\eta)$ plus an absolute constant depending only on $\mathfrak{f}$ ), which is indeed equivalent to (6.1). Clearly, all these requirements on the measure $\mu$ are met in the present case.

Concerning the admissibility property, observe that the zeros of $q_{n}$ are contained in $\mathbb{D}$ by the very definition of $g_{n}$ and their counting measures converge weak $^{*}$ to the Green equilibrium distribution on $[c, d]$ by [9, Thm. 2.1]. Thus, the second requirement for admissibility is satisfied. So, it only remains to construct the rearrangements $\Delta_{n}$ that we shall simply take to be the identity mappings. This way we are required to show that the sums $\sum_{j=1}^{n}\left|\phi\left(\bar{\xi}_{j, n}\right)-\phi\left(\xi_{j, n}\right)\right|$ remain bounded when $n \rightarrow \infty$, where $\xi_{j, n}$ are the zeros of $q_{n}$ and $\phi(\cdot):=\psi(1 / \cdot)$. Since $\phi$ is holomorphic in $\mathfrak{D}^{*},\left\{\xi_{j, n}\right\} \subset \mathbb{D}$, and $\overline{\mathbb{D}} \subset \mathfrak{D}^{*}$, it holds that that

$$
\left|\phi\left(z_{1}\right)-\phi\left(z_{2}\right)\right| \leq s_{1}\left|z_{1}-z_{2}\right|, \quad z_{1}, z_{2} \in \mathbb{D},
$$

where $s_{1}:=\max _{\mathbb{T}}\left|\psi^{\prime}\right|=\max _{\mathbb{T}}\left|\phi^{\prime}\right|=\max _{\mathbb{D}}\left|\phi^{\prime}\right|$ by the very definition of $\phi$ and the maximum modulus principle for analytic functions. Hence,

$$
\sum_{j=1}^{n}\left|\phi\left(\bar{\xi}_{j, n}\right)-\phi\left(\xi_{j, n}\right)\right| \leq 2 s_{1} \sum_{j=1}^{n}\left|\operatorname{Im}\left(\xi_{j, n}\right)\right| \leq 2 s_{1} \sum_{j=1}^{n}\left(\pi-\operatorname{Angle}\left(\xi_{j, n}\right)\right),
$$

where $\operatorname{Angle}(z):=|\operatorname{Arg}(a-z)-\operatorname{Arg}(b-z)| \operatorname{Arg}(z) \in(-\pi, \pi]$ is the principal branch of the argument of $z$, and we set $\operatorname{Arg}(0)=\pi$. The uniform boundedness of the sums on the right-hand side of (6.2) was established in [9, Lem. 3.1], using in an essential manner that the argument of $\dot{\mu}$ is of bounded variation, as a prerequisite for the proof of [9, Thm. 2.1]. This finishes the proof of the lemma. 
Proposition 9. Under the conditions of either Theorem 2 or Theorem 3 it holds that

$$
\left(\mathfrak{f}-g_{n}\right)(z)=\frac{q_{n, m}(z)}{\left(b_{n}^{2} w_{n} q\right)(z)} \int \frac{\left(b_{n}^{2} w_{n} q\right)(t)}{q_{n, m}(t)} \frac{d \mu(t)}{z-t}, \quad z \in \overline{\mathbb{D}} \cap \mathfrak{D} .
$$

Proof. Let $\left\{v_{n}\right\}$ be a sequence of singular vectors associated to $\left\{g_{n}\right\}$ having innerouter factorizations (3.5). It was obtained in [5, Prop. 9.1] that

$$
\begin{array}{ll}
\mathcal{H}_{f}\left(v_{n}\right)(\xi)=\sigma_{n} \bar{\xi} \overline{\left(b_{n} j_{n} w_{n}^{p^{\prime} / 2}\right)(\xi)}=\sigma_{n}\left(b_{n} j_{n} w_{n}^{p^{\prime} / 2}\right)^{\sigma}(\xi), & p>2 \\
\mathcal{H}_{f}\left(v_{n}\right)(\xi)=\bar{\xi} \overline{\left(b_{n} a_{n}\right)(\xi)}=\left(b_{n} a_{n}\right)^{\sigma}(\xi), & p=2,
\end{array}
$$

for a.e. $\xi \in \mathbb{T}$, where $j_{n}$ is some inner function and $a_{n} \in H^{2}$. Following the analysis in [5, Sec. 10], this leads to orthogonality relations of the form

$$
\int \frac{\left(l_{n-1} q_{n} w_{n}\right)(t)}{\widetilde{q}_{n}^{2}(t)} d \mu(t)+\int_{\mathbb{T}} \frac{\left(l_{n-1} q_{n} w_{n}\right)(\tau)}{\widetilde{q}_{n}^{2}(\tau)} \frac{p(\tau)}{q(\tau)} \frac{d \tau}{2 \pi i}=0
$$

for any polynomial $l_{n-1}, \operatorname{deg}\left(l_{n-1}\right) \leq n-1$. In another connection, (3.3) yields that

$$
w_{n}\left(\mathfrak{f}-g_{n}\right)=\frac{w_{n} \mathcal{H}_{\mathfrak{f}}\left(v_{n}\right)}{v_{n}}=\frac{\mathcal{P}_{-}\left(\mathfrak{f} v_{n}\right)}{b_{n}} .
$$

The right-hand side of 6.6 is holomorphic outside of $\overline{\mathbb{D}}$ and is vanishing at infinity. So, by the Cauchy theorem it can be written as

$$
\begin{aligned}
\frac{\mathcal{P}_{-}\left(\mathfrak{f} v_{n}\right)}{b_{n}}(z) & =\frac{1}{b_{n}(z)} \int_{\mathbb{T}} \frac{\left(\mathfrak{f} v_{n}\right)(\tau)}{z-\tau} \frac{d \tau}{2 \pi i} \\
& =\frac{\widetilde{q}_{n}(z)}{q_{n}(z)}\left(\int \frac{\left(q_{n} w_{n}\right)(t)}{\widetilde{q}_{n}(t)} \frac{d \mu(t)}{z-t}+\frac{1}{2 \pi i} \int_{\mathbb{T}} \frac{\left(q_{n} w_{n}\right)(\tau)}{\widetilde{q}_{n}(\tau)} \frac{p(\tau)}{q(\tau)} \frac{d \tau}{z-\tau}\right)
\end{aligned}
$$

for $|z|>1$. Using 6.5 with $l_{n-1}(t)=\left(\widetilde{q}_{n}(z)-\widetilde{q}_{n}(t)\right) /(z-t)$, we get that

$$
\frac{\mathcal{P}_{-}\left(\mathfrak{f} v_{n}\right)}{b_{n}}(z)=\frac{\widetilde{q}_{n}^{2}(z)}{q_{n}(z)}\left(\int \frac{\left(q_{n} w_{n}\right)(t)}{\widetilde{q}_{n}^{2}(t)} \frac{d \mu(t)}{z-t}+\frac{1}{2 \pi i} \int_{\mathbb{T}} \frac{\left(q_{n} w_{n}\right)(\tau)}{\widetilde{q}_{n}^{2}(\tau)} \frac{p(\tau)}{q(\tau)} \frac{d \tau}{z-\tau}\right) .
$$

Applying 6.5 again, now with $l_{n-1}=\left(\left(q u_{n-m}\right)(z)-\left(q u_{n-m}\right)(\cdot)\right) /(z-\cdot)$, and using the Cauchy integral formula to get rid of the second integral, we obtain that

$$
\frac{\mathcal{P}_{-}\left(\mathfrak{f} v_{n}\right)}{b_{n}}(z)=\frac{\widetilde{q}_{n}^{2}(z)}{\left(u_{n-m} q q_{n}\right)(z)} \int \frac{\left(u_{n-m} q q_{n} w_{n}\right)(t)}{\widetilde{q}_{n}^{2}(t)} \frac{d \mu(t)}{z-t}, \quad|z|>1 .
$$

Observe now that the last expression is well-defined as a meromorphic function everywhere in $\mathfrak{D}$. Thus, it follows from 6.6 that 6.3 holds.

Lemma 10. Let $\lambda_{n}$ be a sequence of Borel complex measures on $[c, d]$ such that

$$
\hat{F}_{n}(z):=\int \frac{q(t)}{q_{n, m}(t)} \frac{d \lambda_{n}(t)}{z-t}, \quad z \in \mathfrak{D},
$$

converges to some function $F$ locally uniformly in $\mathfrak{D}$. Then

$$
F_{n}-F=o(1), \quad F_{n}(z):=\int \frac{d \lambda_{n}(t)}{z-t}, \quad z \in \mathfrak{D},
$$


locally uniformly in $\mathfrak{D}$.

Proof. Assume first that $q(z)=(z-\eta)^{m}$. Let $z \in \mathfrak{D}_{\mathfrak{f}}$ and $\Gamma_{1}$ and $\Gamma_{2}$ be two Jordan curves encompassing $[c, d]$ and $\{\eta\}$, respectively, separating them from each other, and containing $z$ within the unbounded components of their complements. Then

$$
\begin{aligned}
\left(\hat{F}_{n} \frac{q_{n, m}}{q}\right)(z) & =\frac{1}{2 \pi i} \int_{\Gamma_{1}}\left(\hat{F}_{n} \frac{q_{n, m}}{q}\right)(\tau) \frac{d \tau}{z-\tau}+\frac{1}{2 \pi i} \int_{\Gamma_{2}}\left(\hat{F}_{n} \frac{q_{n, m}}{q}\right)(\tau) \frac{d \tau}{z-\tau} \\
& =F_{n}(z)+\frac{1}{(m-1) !}\left(\frac{\hat{F}_{n} q_{n, m}}{z-\cdot}\right)^{(m-1)}(\eta),
\end{aligned}
$$

where we used the Fubini-Tonelli theorem and the Cauchy integral formula. Thus,

$$
F_{n}(z)=\left[\left(q_{n, m} \hat{F}_{n}\right)(z)-T_{\eta, m-1}\left(q_{n, m} \hat{F}_{n} ; z\right)\right] / q(z), \quad z \in \mathfrak{D},
$$

where $T_{\eta, m-1}(f ; \cdot)$ is the $(m-1)$-st partial sum of the Taylor expansion of $f$ at $\eta$. By 6.1 the polynomials $T_{\eta, m-1}\left(q_{n, m} \hat{F}_{n} ; \cdot\right)$ converge to zero as $n$ tends to infinity and the claim of the lemma follows by the maximum modulus principle for analytic functions. By partial fraction decomposition, the case of a general $q$ is no different.

Proof of Theorem 1. Let

$$
d \nu_{n}:=\frac{q_{n+m, m} q w_{n+m}}{\widetilde{q}_{n+m}^{2}} d \mu=\frac{q_{n+m, m} q w_{n+m} h \hbar}{\widetilde{q}_{n+m}^{2}} d \omega_{[c, d]} .
$$

Then we get from 6.5 applied with $n$ replaced by $n+m$ and $l_{n+m-1}(t)=t^{j} q(t)$, $j=0, \ldots, n-1$, that

$$
\int t^{j} u_{n}(t) d \nu_{n}(t)=0
$$

So, the asymptotic behavior of $u_{n}$ is governed by Theorem 7 , applied with $v_{n}=\widetilde{q}_{n}^{2}$ and $h_{n}=q_{n+m, m} q w_{n+m}$, due to Lemma 8 and the fact that $\left\{w_{n}\right\}$ is a normal family in $\mathbb{D}$ none of which limit points has zeros. The latter was obtained in [9, Lem. 3.4] under the mere assumption that $\mu$ has infinitely many points in the support and an argument of bounded variation.

In another connection, observe that

$$
b_{n}\left(b_{n} j_{n} w_{n}^{p^{\prime} / 2}\right)^{\sigma}=\left|b_{n}\right|^{2}\left(j_{n} w_{n}^{p^{\prime} / 2}\right)^{\sigma}=\left(j_{n} w_{n}^{p^{\prime} / 2}\right)^{\sigma} \quad \text { on } \quad \mathbb{T}
$$

and that $\left(j_{n} w_{n}^{p^{\prime} / 2}\right)^{\sigma}$ is the trace of a function from $\bar{H}_{0}^{2}$. Thus, it follows from 6.4 that

$$
\mathcal{P}_{-}\left(b_{n} \mathcal{H}_{\mathfrak{f}}\left(v_{n}\right)\right)=\sigma_{n} \mathcal{P}_{-}\left(b_{n}\left(b_{n} j_{n} w_{n}^{p^{\prime} / 2}\right)^{\sigma}\right)=\sigma_{n}\left(j_{n} w_{n}^{p^{\prime} / 2}\right)^{\sigma} .
$$

It is also readily checked that

$$
\mathcal{P}_{-}\left(b_{n} \mathcal{H}_{\mathfrak{f}}\left(v_{n}\right)\right)(z)=\mathcal{P}_{-}\left(b_{n} \mathcal{P}_{-}\left(\mathfrak{f} v_{n}\right)\right)(z)=\mathcal{P}_{-}\left(\mathfrak{f} b_{n} v_{n}\right)(z)=\int_{\mathbb{T}} \frac{\left(\mathfrak{f} b_{n} v_{n}\right)(\tau)}{z-\tau} \frac{d \tau}{2 \pi i}
$$


for $|z|>1$. Hence, we derive by using the Fubini-Tonelli theorem that

$$
\frac{\sigma_{n}}{\gamma_{n-m}}\left(j_{n} w_{n}^{p^{\prime} / 2}\right)^{\sigma}(z)=\frac{1}{\gamma_{n-m}}\left(\int \frac{\left(b_{n}^{2} w_{n}\right)(t)}{z-t} d \mu(t)+\int_{\mathbb{T}} \frac{\left(b_{n}^{2} w_{n}\right)(\tau)}{z-\tau} \frac{p(\tau)}{q(\tau)} \frac{d \tau}{2 \pi i}\right),
$$

for $|z|>1$, where $\gamma_{n}$ has the same meaning as in Theorem 7 As the right-hand side of $(6.10)$ is defined everywhere in $\mathfrak{D}_{\mathfrak{f}}$, the restriction $|z|>1$ is no longer necessary. This, in particular, implies that $j_{n}$ is a finite Blaschke product as neither singular inner factors nor infinite Blaschke products can be extended even continuously on $\mathbb{T}$. However, notice that first we should evaluate the second integral on the righthand side of $(6.10)$ by the residue formula and only then remove the restriction $|z|>1$. Clearly, this integral represents a rational function vanishing at infinity whose poles are those of $q$. It is also easy to observe that if

$$
\gamma_{n-m}^{-1}\left(b_{n}^{2} w_{n} p\right)^{(k)}(\eta)=o(1), \quad k=0, \ldots, m(\eta)-1, \quad \text { and } \quad\left(b_{n}^{2} w_{n} p\right)(\eta) \neq 0,
$$

this rational function converges to zero locally uniformly in $\overline{\mathbb{C}} \backslash z(q)$ and has poles of exact multiplicity $m(\eta)$ at each $\eta \in \mathrm{z}(q)$. Now, we have by (6.3), 5.2), and (5.3) that

$$
\left(\mathfrak{f}-g_{n}\right)=\frac{q_{n, m}}{q} \frac{\gamma_{n-m} y_{n}}{b_{n}^{2} w_{n}}, \quad y_{n}:=\frac{1}{\gamma_{n-m}} u_{n-m} R_{n-m},
$$

and

$$
y_{n}(z):=\int \frac{u_{n-m}^{2}(t)}{\gamma_{n-m}} \frac{d \nu_{n-m}(t)}{z-t}=\int \frac{\left(b_{n}^{2} w_{n}\right)(t)}{\gamma_{n-m}} \frac{q(t)}{q_{n, m}(t)} \frac{d \mu(t)}{z-t}=\frac{1+o(1)}{\mathfrak{w}(z)}
$$

locally uniformly in $\mathfrak{D}$. Then we get from 6.12 that

$$
\left(q_{n, m} y_{n}\right)(z)=\gamma_{n-m}^{-1}\left(b_{n}^{2} w_{n}\right)(z)\left(\mathfrak{f}_{\mu} q+p\right)(z)+\gamma_{n-m}^{-1}\left(q b_{n} w_{n} h_{n}\right)(z), \quad z \in \mathfrak{D},
$$

where $\mathfrak{f}=\mathfrak{f}_{\mu}+p / q$ and $g_{n}=h_{n} / b_{n}$, and therefore for $\eta \in \mathrm{z}(q)$ we obtain

$$
\left(q_{n, m} y_{n}\right)^{(k)}(\eta)=\gamma_{n-m}^{-1}\left(b_{n}^{2} w_{n} p\right)^{(k)}(\eta), \quad k=0, \ldots, m(\eta)-1 .
$$

Hence, the first part of (6.11) follows from (6.1) and the normality of $\left\{y_{n}\right\}$, which is immediately deduced from 6.13). The second part of 6.11 holds since 6.13) and (6.14), applied with $k=0$, yield that for all $n$ large enough we have

$$
\left.0 \neq y_{n}(\eta)=\gamma_{n-m}^{-1}\left(b_{n} w_{n} p u_{n-m} / \widetilde{q}_{n}\right)\right)(\eta)
$$

and therefore $\left(b_{n} w_{n} p\right)(\eta)$ as well as $\left(b_{n}^{2} w_{n} p\right)(\eta)$ cannot vanish.

In another connection, 6.13 and Lemma 10 yield that

$$
\frac{1}{\gamma_{n-m}} \int \frac{\left(b_{n}^{2} w_{n}\right)(t)}{z-t} d \mu(t)=\frac{1+o(1)}{\mathfrak{w}(z)}
$$

locally uniformly in $\mathfrak{D}$. Thus, combining 6.11) and 6.15) with 6.10, we get that

$$
\frac{\sigma_{n}}{\gamma_{n-m}}\left(j_{n} w_{n}^{p^{\prime} / 2}\right)^{\sigma}=\frac{1+o(1)}{\mathfrak{w}}+\frac{\ell_{n}}{q}
$$


locally uniformly in $\mathfrak{D}$, where $\operatorname{deg}\left(\ell_{n}\right)<m$, the polynomials $\ell_{n}$ are coprime with $q$, and converge to zero locally uniformly in $\mathbb{C}$ when $n \rightarrow \infty$. Equivalently, we have that

$$
\frac{\sigma_{n}}{\bar{\gamma}_{n-m}} j_{n} w_{n}^{p^{\prime} / 2} \widetilde{\mathfrak{w}}=1+o(1)+\frac{\tilde{\ell}_{n} \widetilde{\mathfrak{w}}}{\widetilde{q}}=1+o(1),
$$

where the first $o(1)$ holds locally uniformly in $\mathfrak{D}^{*}$, the second one holds locally uniformly in $\mathfrak{D}_{\mathfrak{f}}^{*}$, and $\tilde{\ell}_{n}(z):=z^{m-1} \overline{\ell_{n}(1 / \bar{z})}, \operatorname{deg}\left(\widetilde{\ell}_{n}\right)<m$ since $\operatorname{deg}\left(\ell_{n}\right)<m$. This, in particular, implies that the Blaschke products $j_{n}$ are identically 1 for all $n$ large enough since the right-hand side of 6.16 cannot vanish in $\overline{\mathbb{D}}$ for such $n$. Finally, recall that by its very definition $w_{n}^{p^{\prime} / 2}$ has unit $L^{2}$ norm. Therefore, deformation of the integral on $\mathbb{T}$ to $[c, d]$ covered twice by the Cauchy integral formula yields that

$$
\begin{aligned}
\left(\frac{\sigma_{n}}{\left|\gamma_{n-m}\right|}\right)^{2} & =\frac{1}{2 \pi} \int_{\mathbb{T}} \frac{|d \tau|}{|\widetilde{\mathfrak{w}}(\tau)|^{2}}+o(1)=\frac{1}{2 \pi i} \int_{\mathbb{T}} \frac{d \tau}{(\mathfrak{w} \widetilde{\mathfrak{w}})(\tau)}+o(1) \\
& =\frac{1}{\pi i} \int_{[c, d]} \frac{d t}{\left(\mathfrak{w}^{-\widetilde{\mathfrak{w}}}\right)(t)}+o(1)=\mathcal{T}^{-2}+o(1)
\end{aligned}
$$

since $\widetilde{\mathfrak{w}}(\tau)=\tau \overline{\mathfrak{w}(\tau)}$ on $\mathbb{T}, \mathfrak{w}^{-}=-\mathfrak{w}^{+}=-i\left|\mathfrak{w}^{ \pm}\right|$on $[c, d]$, and on account of 2.15. Thus, 3.6 shall follow from (6.16) and 6.17) with $l_{n}:=\mathcal{T} \widetilde{\ell}_{n}$ upon showing that

$$
\gamma_{n} /\left|\gamma_{n}\right|=1+o(1) \text {. }
$$

The latter is an easy consequence of $(6.16)$ since $\tilde{\mathfrak{w}}(0)=1$ and $w_{n}(0)>0$.

In the next proposition of technical nature, we define a special sequence of Szegö functions for the condenser $\mathfrak{D} \cap \mathfrak{D}^{*}$ that appears in Theorem 2 .

Proposition 11. For each $p \in[2, \infty]$ there exists a normal family of non-vanishing functions in $\mathfrak{D} \cap \mathfrak{D}^{*}$, denoted by $\left\{D_{n}\right\}$, such that

$$
\hat{M}\left|D_{n}^{ \pm}\right|=\left|\left(r_{n} / r\right)^{ \pm} S_{b^{2} w \dot{\mu}}^{ \pm}\right| \quad \text { on } \quad[c, d], \quad G_{\widetilde{q}_{n} / \widetilde{q}} \frac{\left(S_{b^{2} w \dot{\mu}} r_{n}\right)(1)}{\left(r D_{n}\right)(1)}>0
$$

where $r_{n}:=r_{n}\left(\widetilde{q}_{n} ; \cdot\right), r:=r_{m}(\widetilde{q} ; \cdot), \hat{M}^{2}=M /\left|G_{b^{2} w \dot{\mu}}\right|$, and $M$ is given by 3.10 . Moreover, each $D_{n}$ satisfies $D_{n}(z) \overline{D_{n}(1 / \bar{z})}=1, z \in \mathfrak{D} \cap \mathfrak{D}^{*}$, has continuous traces on each side of $[c, d]$, and has winding number zero on any curve separating $[c, d]$ from $[c, d]^{*}$.

Proof. The concept of Szegö function for a condenser initially was developed in [16. Thm. 1.6] in the case of an annulus. It was shown that if $Y$ is a continuous (strictly) positive function on $\mathbb{T}_{s}, s<1$, then there exists a function $u$, harmonic in $\mathbb{A}_{s}$, such that $u=\frac{1}{2} \log \left(Y / M_{Y}\right)$ on $\mathbb{T}_{s}, u \equiv 0$ on $\mathbb{T}$, and $u(1 / \bar{z})=-u(z), z \in \mathbb{A}_{s}$, where $M_{Y}:=\exp \left\{\int_{\mathbb{T}_{s}} \log Y(\tau) \frac{|d \tau|}{2 \pi s}\right\}$ is the geometric mean of $Y$. Moreover, it was shown that $u$ has single-valued harmonic conjugate $v$. Moreover, the latter is unique up to an additive constant. Finally, it was deduced that $D_{s}(Y ; \cdot):=\exp \{u+i v\}$, the Szegö function of $Y$ for $\mathbb{A}_{s}$, is a non-vanishing holomorphic function in $\mathbb{A}_{s}$ such 
that $M_{Y}\left|D_{s}(Y ; \cdot)\right|^{2}=Y$ on $\mathbb{T}_{s}, D_{s}(Y ; z) \overline{D_{s}(Y ; 1 / \bar{z})}=1, z \in \mathbb{A}_{s}$, and $D_{s}(Y ; \cdot)$ is an outer function in $\mathbb{A}_{s}$ with zero winding number on any curve in $\mathbb{A}_{s}$. The latter was not explicitly stated in [16] but clearly holds since $\log \left|D_{s}(Y ; \cdot)\right|=u$ and therefore it is the integral of its boundary values against the harmonic measure on $\partial \mathbb{A}_{s}$ while $\operatorname{Arg}\left(D_{s}(Y ; \cdot)\right)=v$, which has zero increment on any curve in $\mathbb{A}_{s}$. Obviously, the Szegö function for $\mathbb{A}_{s}$ is unique up to a multiplicative unimodular constant.

Let now $y^{+}$and $y^{-}$be two continuous positive functions on $[c, d]$ whose values at the endpoints coincide. We define the geometric mean and the Szegö function of the pair $\mathfrak{y}:=\left(y^{+}, y^{-}\right)$for the condenser $\mathfrak{D} \cap \mathfrak{D}^{*}$ by

$$
M_{\mathfrak{y}}:=M_{Y} \quad \text { and } \quad D_{\mathfrak{y}}(z):=D_{\rho}(Y ; \varphi(z)), \quad z \in \mathfrak{D} \cap \mathfrak{D}^{*},
$$

respectively, where $Y(\tau)=y^{ \pm}\left(\varphi^{-1}(\tau)\right), \tau \in \mathbb{T}_{\rho}^{ \pm}$. It is an immediate consequence of the corresponding properties of $D_{\rho}(Y ; \cdot)$ and $\varphi$ that $D_{\mathfrak{y}}$ is outer, has non-tangential continuous boundary values on both sides of $[c, d]$ and $[c, d]^{*}$ whenever $\mathfrak{y}$ is a Dinicontinuous pain has winding number zero on any curve in $\mathfrak{D} \cap \mathfrak{D}^{*}$, and satisfies

$$
\left|D_{\mathfrak{y}}^{ \pm}(t)\right|^{2}= \begin{cases}y^{ \pm}(t) / M_{\mathfrak{y}}, & t \in[c, d] \\ M_{\mathfrak{y}} / y^{ \pm}(1 / t), & t \in[c, d]^{*}\end{cases}
$$

and $D_{\mathfrak{y}}(z) \overline{D_{\mathfrak{y}}(1 / \bar{z})}=1, z \in \mathfrak{D} \cap \mathfrak{D}^{*}$.

Now, put $y^{ \pm}:=\left|b^{2} w \dot{\mu}\right|$ and $y_{n}^{ \pm}:=\left|\left(r_{n} / r\right)^{ \pm} S_{b^{2}}^{ \pm} w \dot{\mu}\right|^{2}$. Observe that in this case

$$
y_{n}^{+}(t) y_{n}^{-}(t)=\left|S_{b^{2} w \dot{\mu}}^{+}(t) S_{b^{2} w \dot{\mu}}^{-}(t)\right|^{2}=\left|\frac{\left(b^{2} w \dot{\mu}\right)(t)}{G_{b^{2} w \dot{\mu}}}\right|^{2}=\frac{y^{+}(t) y^{-}(t)}{\left|G_{b^{2} w \dot{\mu}}\right|^{2}},
$$

$t \in[c, d]$, by (2.7) and (2.9). Then for $\mathfrak{y}_{n}:=\left(y_{n}^{+}, y_{n}^{-}\right)$we get that

$$
\begin{aligned}
\log M_{\mathfrak{y}_{n}} & =\int_{\mathbb{T}_{\rho}^{+}} \log \left[Y_{n}(\tau) Y_{n}(\bar{\tau})\right] \frac{|d \tau|}{2 \pi \rho}=\int_{\mathbb{T}_{\rho}^{+}} \log \left[y_{n}^{+}(t) y_{n}^{-}(t)\right] \frac{|d \tau|}{2 \pi \rho} \\
& =\int_{\mathbb{T}_{\rho}^{+}} \log \left[\frac{y^{+}(t) y^{-}(t)}{\left|G_{b^{2} w \dot{\mu}}\right|^{2}}\right] \frac{|d \tau|}{2 \pi \rho}=\log M_{\mathfrak{y}}-\log \left|G_{b^{2} w \dot{\mu}}\right|,
\end{aligned}
$$

where $t=\varphi^{-1}(\tau)=\varphi^{-1}(\bar{\tau})$. Further, by 2.15) and (3.10), we have that

$$
\log M_{\mathfrak{y}}=\int_{[c, d]} \log \left|\left(b^{2} w \dot{\mu}\right)(t)\right| \frac{d\left|\varphi^{+}(t)\right|}{\pi \rho}=\log M .
$$

So, $M_{\mathfrak{y}_{n}}=M /\left|G_{b^{2} w \dot{\mu}}\right|=\hat{M}^{2}$ and therefore the claim of the proposition follows by setting $D_{n}:=D_{\mathfrak{y}_{n}}$ with the chosen normalization (recall that the functions $D_{\mathfrak{y}_{n}}$ are uniquely defined up to a unimodular constant).

The following Lemma was proved in [6, Lem. 4.7].

\footnotetext{
${ }^{4}$ This means that $Y$ and therefore $\log Y$ are Dini-continuous as $\varphi^{-1}$ is Lipschitz on $\mathbb{T}_{\rho}$. Hence, the boundary values of $v$ are continuous on $\partial \mathbb{A}_{\rho}$ [12, Ch. III].
} 
Lemma 12. Let $U$ be a domain, $\partial U=K_{1} \cup K_{2}, K_{1}$ and $K_{2}$ be two disjoint compact sets in $\mathbb{C}$, and $u$ be a harmonic function in $U$. Assume that

$$
\int_{\Gamma} \frac{\partial u}{\partial n} d s=0
$$

where $\partial / \partial n$ and $d s$ are, respectively, the normal derivative and the arclength differential on $\Gamma$, and the latter is an oriented smooth Jordan curve that separates $K_{1}$ from $K_{2}$, has winding number 1 with respect to any point of $K_{1}$, and winding number 0 with respect to any point of $K_{2}$. Then

$$
\sup _{z^{\prime} \in K_{1}} \limsup _{z \rightarrow z^{\prime},} u(z) \geq \inf _{z^{\prime} \in K_{2}} \liminf _{z \rightarrow z^{\prime}, z \in U} u(z)
$$

and the same relation holds with $K_{1}$ and $K_{2}$ interchanged.

Proof of Theorem 2. It was shown in Lemma 8 that $q_{n}$ can be written as $u_{n-m} q_{n, m}$ and the behavior of $u_{n-m}$ is governed by Theorem 7 with $\nu_{n}$ defined in (6.8). Thus, we have from (5.3) that

$$
1+o(1)=u_{n-m} S_{n-m}=u_{n-m}(\kappa \psi)^{n-m} S_{q \dot{\mu} / \widetilde{q}_{n}^{2}} S_{q_{n, m} w_{n}}
$$

locally uniformly in $\mathfrak{D}$. Further, since $q_{n, m} w_{n}$ converges to $q w$ uniformly on $[c, d]$ by Lemma 8 and Theorem 1 we get that $S_{q_{n, m} w_{n}}=[1+o(1)] S_{q w}$ uniformly in $\mathfrak{D}$ and therefore we obtain from 6.19 that

$$
1+o(1)=u_{n-m} \kappa^{n-m} S_{b^{2} w \dot{\mu}} \psi^{n-m} S_{\widetilde{q}^{2} / \widetilde{q}_{n}^{2}}
$$

locally uniformly in $\mathfrak{D}$, where $b^{2}=q^{2} / \widetilde{q}^{2}$. Now, it follows from 2.10 that

$$
S_{\widetilde{q}^{2} / \widetilde{q}_{n}^{2}}=S_{\widetilde{q} / \widetilde{q}_{n}}^{2}=\frac{\psi^{m-n}}{G_{\widetilde{q} / \widetilde{q}_{n}}} \frac{\widetilde{q}}{\widetilde{q}_{n}} \frac{r_{n}}{r},
$$

where $r:=r_{m}(\widetilde{q} ; \cdot)$ and $r_{n}:=r_{n}\left(\widetilde{q}_{n} ; \cdot\right)$ as in Proposition 11. Hence, we deduce from 6.20 that

$$
1+o(1)=\frac{b_{n}}{b} \frac{q}{q_{n, m}} \frac{\kappa^{n-m}}{G_{\widetilde{q} / \widetilde{q}_{n}}} S_{b^{2} w \dot{\mu}} \frac{r_{n}}{r}
$$

locally uniformly in $\mathfrak{D}$. Then Lemma 8 implies that

$$
\lambda_{n} X_{n} D_{n} b_{n} \varphi^{m-n} b^{-1}=1+o(1),
$$

locally uniformly in $\mathfrak{D}_{\mathfrak{f}}$, where

$$
\lambda_{n}:=\frac{\hat{M}(\kappa \rho)^{n-m}}{G_{\widetilde{q} / \widetilde{q}_{n}}} \quad \text { and } \quad X_{n}(z):=\frac{S_{b^{2} w \dot{\mu}}}{\hat{M} D_{n}} \frac{r_{n}}{r}\left(\frac{\varphi}{\rho}\right)^{n-m} .
$$

Now, we shall show that

$$
\lambda_{n} X_{n}=1+o(1) \quad \text { uniformly in } \overline{\mathbb{D}} \text {. }
$$

Observe, that

$$
\left|X_{n}^{ \pm}\right|=\left|\left(\frac{S_{b^{2} w \dot{\mu}}}{\hat{M} D_{n}} \frac{r_{n}}{r}\right)^{ \pm}\right| \equiv 1 \quad \text { on } \quad[c, d]
$$


by the very definition of $D_{n}$ (see Proposition 11). Moreover, since the zeros of $r, \mathrm{z}(\widetilde{q})$, lie outside of $\overline{\mathbb{D}}$ and the zeros of $r_{n}, \mathrm{z}\left(\widetilde{q}_{n}\right)$, approach $[c, d]^{*}$ and $\mathrm{z}(\widetilde{q})$ by Lemma 8 and Theorem 7 , the functions $X_{n}$ are zero free in some neighborhood of $\overline{\mathbb{D}}$, where the values on $[c, d]$ are twofold. Further, the winding number of $X_{n}$ along any smooth Jordan curve encompassing $[c, d]$ in $\mathbb{D}$ is equal to zero. Indeed, the winding number of $S_{b^{2} w \dot{\mu}} / D_{n}$ on such a curve is zero by the properties of Szegö functions, $r_{n} / r$ has winding number $m-n$ since it is meromorphic outside of $[c, d]$ with $n$ zeros and $m$ poles outside of $\overline{\mathbb{D}}$, and it follows from [18, Ch. VI] that $\varphi$ has winding number one on any such curve. Thus, $\log X_{n}$ are well-defined holomorphic functions in $\overline{\mathbb{D}} \backslash[c, d]$. In turn, this means that $\log \left|X_{n}\right|$ satisfies the conditions of Lemma 12 with $U=\mathbb{D} \backslash[c, d]$. Applying this lemma in both directions, we get from $(6.24)$ that

$$
\inf _{\mathbb{T}}\left|X_{n}\right| \leq \sup _{[c, d]}\left|X_{n}^{ \pm}\right|=1=\inf _{[c, d]}\left|X_{n}^{ \pm}\right| \leq \sup _{\mathbb{T}}\left|X_{n}\right| .
$$

In another connection, 6.21 and 6.1 yield that uniformly on $\mathbb{T}$ we have

$$
\left|X_{n}\right|=\left|\frac{G_{\widetilde{q} / \widetilde{q}_{n}}}{\hat{M}(\kappa \rho)^{n-m}} \frac{\kappa^{n-m}}{G_{\widetilde{q} / \widetilde{q}_{n}}} S_{b^{2} w \dot{\mu}} \frac{r_{n}}{r}\right|=\frac{1+o(1)}{\left|\lambda_{n}\right|}
$$

since $D_{n}, \varphi, b_{n}$, and $b$ are unimodular on $\mathbb{T}$. Combining 6.25) with 6.26), we get that $\left|\lambda_{n}\right|=1+o(1)$ and therefore

$$
\left|\lambda_{n} X_{n}\right|=\left|\frac{(\kappa \varphi)^{n-m}}{G_{\widetilde{q} / \widetilde{q}_{n}}} \frac{S_{b^{2} w \dot{\mu}} r_{n}}{D_{n} r}\right|=1+o(1) \text { uniformly in } \overline{\mathbb{D}}
$$

by the maximum principle for harmonic functions applied to $\pm \log \left|X_{n}\right|$ in $\mathbb{D} \backslash[c, d]$. Hence, $\left\{\lambda_{n} X_{n}\right\}$ is a normal family of harmonic functions in $\mathbb{D} \backslash[c, d]$ and all the limit points of this family are the unimodular constants. Therefore 6.23 follows from the normalization of $D_{n}$ (see Proposition 11) and the fact that $\varphi(1)=1$.

Clearly, we can rewrite 6.22 with the help of 6.23 as

$$
D_{n} b_{n} \varphi^{m-n} b^{-1}=1+o(1) \quad \text { uniformly on compact subsets of } \quad \overline{\mathbb{D}} \cap \mathfrak{D}_{\mathfrak{f}} .
$$

Now, recall that $\overline{D_{n}(1 / \cdot)}=1 / D_{n}$. Moreover, the same property holds for $b_{n}, b$, and $\varphi$. Thus,

$$
D_{n} b_{n} \varphi^{m-n} b^{-1}=1 / \overline{\left(D_{n} b_{n} \varphi^{m-n} b^{-1}\right)(1 / \cdot)}=1+o(1)
$$

uniformly on closed subsets of $\mathfrak{D}_{f}^{*} \backslash \mathbb{D}$ and 3.7 follows.

It only remains to prove (3.8) and $(3.9)$. By the very definition of $\gamma_{n}$ in Theorem 7, we have that

$$
\left|\gamma_{n-m}\right|=\frac{2\left|G_{\dot{\nu}_{n-m}}\right|}{\kappa^{2(n-m)}}=[1+o(1)] \frac{2\left|G_{b^{2} w \dot{\mu}}\right|\left|G_{\widetilde{q} / \widetilde{q}_{n}}^{2}\right|}{\kappa^{2(n-m)}}
$$

by Lemma 8 and limit 3.6 . Further, the very definitions of $\hat{M}$ and $\lambda_{n}$ yield that

$$
\frac{2\left|G_{b^{2} w \dot{\mu}}\right|\left|G_{\widetilde{q} / \widetilde{q}_{n}}^{2}\right|}{\kappa^{2(n-m)}}=\frac{2\left|G_{b^{2} w \dot{\mu}}\right| \hat{M}^{2}}{\rho^{2(m-n)}} \frac{\left|G_{\widetilde{q} / \widetilde{q}_{n}}\right|^{2}}{\hat{M}^{2}(\kappa \rho)^{2(n-m)}}=\frac{2 M \rho^{2(n-m)}}{\left|\lambda_{n}\right|^{2}} .
$$


Since $\left|\lambda_{n}\right|=1+o(1)$, it holds that

$$
\gamma_{n-m}=[1+o(1)]\left|\gamma_{n-m}\right|=[1+o(1)] 2 M \rho^{2(n-m)},
$$

where we used (6.18). Thus, (3.8) follows from 6.17). Finally, we deduce from 6.12 and 6.13 that

$$
\left(\mathfrak{f}-g_{n}\right)=[1+o(1)] \frac{q_{n, m}}{q} \frac{\gamma_{n-m}}{b_{n}^{2} w_{n}} \frac{1+o(1)}{\mathfrak{w}}
$$

uniformly on compact subsets of $\mathfrak{D}_{\mathfrak{f}} \cap \overline{\mathbb{D}}$. Since $q_{n, m} / q \rightarrow 1$ by Lemma $8, w_{n} \rightarrow w$ by 3.6 , and using 6.28 with 3.7 , 3.9 follows.

Proof of Theorem 3. Recall that Lemma 8 holds under the conditions of this theorem and the Szegö functions $D_{n}$ exist for $p=2$ as well. As the starting point of the proof of Theorem 2 was the application of (5.3) with $\nu_{n}$ defined in (6.8), all we need to do is to show that the conditions of Theorem 7 still hold under the present assumptions. This is tantamount to show that $\Upsilon_{x}$, defined in (5.1), is minorized by $\Psi_{x}$, defined in the statement of the theorem, for all $x \in \mathbf{x}$. In other words, that

$$
\Psi_{x} \leq \liminf _{n \rightarrow \infty} \min _{ \pm}\left\{\left|\left(r_{n} Q_{q q_{n, m} h}\right)^{ \pm}(x)\right|\right\}, \quad x \in \mathbf{x} .
$$

Equivalently, we need to show that

$$
-\liminf _{n \rightarrow \infty} \log \left|r_{n}^{ \pm}(x)\right|^{2}=\limsup _{n \rightarrow \infty} \log \left|r_{n}^{\mp}(x)\right|^{2} \leq \frac{4 s_{1}\left(V_{h}+2 m \pi\right)}{1-s_{0}}, \quad x \in \mathbf{x},
$$

by Lemma 8 and the definition of $\Psi_{x}$. Let, as in the proof of Lemma $8, \xi_{j, n}$, $j=1, \ldots, n$, be the zeros of $q_{n}$. Then we get from 6.2 that

$$
\sum_{j=1}^{n}\left|\psi\left(1 / \bar{\xi}_{j, n}\right)-\psi\left(1 / \xi_{j, n}\right)\right| \leq 2 s_{1} \sum_{j=1}^{n}\left(\pi-\operatorname{Angle}\left(\xi_{j, n}\right)\right) \leq 2 s_{1}\left(V_{h}+2 m \pi\right),
$$

where we used [9, Lem. 3.2] for the last inequality. Put $\hat{r}_{n}:=r_{n} \circ \psi^{-1}$. Then, exactly as we did to prove (5.4), we obtain that

$$
\log \left|\hat{r}_{n}\right| \leq 2 s_{1}\left(V_{h}+2 m \pi\right) /\left(1-s_{0}\right)
$$

which finishes the proof of the theorem.

To prove Theorem 4 we need the following lemma.

Lemma 13. Let $R=P / Q$ be a rational function of degree $d, \zeta \in \mathbb{C}$, and $\delta>0$. Assume further that $P$ and $Q$ have no zeros in $\{z:|z-\zeta| \leq \delta\}$. Then for any $k<d, k \in \mathbb{N}$, there exists $c_{k}=c_{k}(\delta)$ independent of $R$ such that

$$
\left|R^{(k)}(\zeta) / R(\zeta)\right| \leq c_{k} d^{k}
$$

Proof. Clearly, if $T$ is a polynomial of degree at most $d$ with no zeros in $\{z$ : $|z-\zeta| \leq \delta\}$, then

$$
\left|\frac{T^{(j)}(\zeta)}{T(\zeta)}\right| \leq \frac{d \cdot \ldots \cdot(d-j+1)}{\delta^{j}} \leq\left(\frac{d}{\delta}\right)^{j}, \quad j=1, \ldots, k .
$$


Thus, it can be checked that

$$
\left|T(\zeta)\left(\frac{1}{T(\zeta)}\right)^{(j)}\right|=\left|\sum_{l=1}^{j} \sum_{\sum d_{i}=l} \prod_{\sum s_{i} d_{i}=j} c_{l,\left\{d_{i}\right\},\left\{s_{i}\right\}}\left(\frac{T^{\left(s_{i}\right)}(\zeta)}{T(\zeta)}\right)^{d_{i}}\right| \leq c_{j}^{*} d^{j},
$$

$j=1, \ldots, k$, where coefficients $c_{l,\left\{d_{i}\right\},\left\{s_{i}\right\}}$ do not depend on $T$. Then

$$
\left|\frac{R^{(k)}(\zeta)}{R(\zeta)}\right|=\left|\sum_{j=1}^{k}\left(\begin{array}{c}
k \\
j
\end{array}\right) \frac{P^{(j)}(\zeta)}{P(\zeta)} Q(\zeta)\left(\frac{1}{Q(\zeta)}\right)^{(k-j)}\right| \leq \sum_{j=1}^{k}\left(\begin{array}{c}
k \\
j
\end{array}\right) \frac{c_{k-j}^{*} d^{k}}{\delta^{j}}=: c_{k} d^{k}
$$

Proof of Theorem 4. As the forthcoming analysis is local around $\eta$, we may suppose without loss of generality that $m(\eta)=m$, i.e. $\eta$ is the only zero of $q$.

Exactly as in 6.14, we obtain that

$$
y_{n}^{(k)}(\eta)=\left(Y_{n} q_{n, m}\right)^{(k)}(\eta), \quad k=0, \ldots, m-1, \quad Y_{n}:=\frac{u_{n-m}^{2} w_{n} p}{\gamma_{n-m} \widetilde{q}_{n}^{2}} .
$$

It is apparent from 6.22, 6.23, and foremost 6.21, which holds locally uniformly in $\mathfrak{D}$ rather then $\mathfrak{D}_{\mathfrak{f}}$, that

$$
\frac{u_{n-m}}{\widetilde{u}_{n-m}} \frac{D_{n}}{\varphi^{n-m}}=1+o(1) \quad \text { locally uniformly in } \quad \mathbb{D} \cap \mathfrak{D} \text {. }
$$

So, we see using (3.6), 6.1), 6.17) with 6.18), and (3.8) that

$$
Y_{n}=[1+o(1)] \frac{\varphi^{2(n-m)}}{\mathcal{T} \sigma_{n}} \frac{w p}{D_{n}^{2} \widetilde{q}^{2}}=\left[\frac{1}{2 M}+o(1)\right]\left(\frac{\varphi}{\rho}\right)^{2(n-m)} \frac{w p}{D_{n}^{2} \widetilde{q}^{2}}
$$

uniformly in some neighborhood of $\eta$. Thus, we obtain from 6.29 with $k=0$ that

$$
\chi_{n}^{m} q_{n, m}(\eta)=-1, \quad \chi_{n}^{m}:=-Y_{n}(\eta) / y_{n}(\eta),
$$

where for each $n$ we fixed an arbitrary root $\chi_{n}$. Observe also that $\chi_{n}$ tends to infinity geometrically fast by $(6.30)$ since $|\varphi(\eta)|>\rho$ and the boundedness of $\left\{\left|y_{n}(\eta)\right|\right\}$, which is apparent from 6.13). By putting $k=1$ in 6.29), we see that

$$
\chi_{n}^{m-1} q_{n, m}^{\prime}(\eta)=\frac{1}{\chi_{n}}\left(\frac{Y_{n}^{\prime}(\eta)}{Y_{n}(\eta)}-\frac{y_{n}^{\prime}(\eta)}{y_{n}(\eta)}\right)=o(1)
$$

since $\left\{y_{n}^{\prime}(\eta) / y_{n}(\eta)\right\}$ is a convergent sequence by 6.13) and $Y_{n}$ are rational functions, which do not vanish in some fixed neighborhood of $\eta$, multiplied by $w_{n}$, which form a convergent sequence by $(3.6)$, the numbers $\left|\left(Y_{n}^{\prime} / Y_{n}\right)(\eta)\right|$ grow linearly with $n$ by Lemma 13 while $1 / \chi_{n}$ decays exponentially. Continuing by induction, we get

$$
\chi_{n}^{m-k} q_{n, m}^{(k)}(\eta)=\sum_{j=1}^{k}\left(\begin{array}{c}
k \\
j
\end{array}\right) \frac{Y_{n}^{(j)}(\eta)}{Y_{n}(\eta)} \frac{\chi_{n}^{m-k+j} q_{n, m}^{(k-j)}(\eta)}{\chi_{n}^{j}}-\frac{1}{\chi_{n}^{k}} \frac{y_{n}^{(k)}(\eta)}{y_{n}(\eta)}=o(1),
$$


for any $k=2, \ldots, m-1$. Hence, we deduce from 6.31 and 6.32 that

$$
\prod_{k=1}^{m}\left(z+\chi_{n}\left(\eta-\eta_{k, n}\right)\right)=z^{m}+\sum_{k=0}^{m-1} \chi_{n}^{m-k} q_{n, m}^{(k)}(\eta) z^{k}=z^{m}+o(1)-1,
$$

uniformly in some neighborhood of $\eta$. In particular, this means that

$$
\eta_{k, n}=\eta+\frac{1+\delta_{k, n}}{\chi_{n}} \exp \left\{\frac{2 \pi k i}{m}\right\}, \quad k=1, \ldots, m,
$$

where $\delta_{k, n}=o(1)$ for each $k$ and $\prod_{k=1}^{m}\left(1+\delta_{k, n}\right)=1$. By setting

$$
\left(A_{k, n}^{\eta}\right)^{m}:=\frac{1+\delta_{k, n}}{\chi_{n}^{m}}\left(\frac{\rho}{\varphi(\eta)}\right)^{2(m-n)}=-\left[1+\delta_{k, n}\right]\left(\frac{\rho}{\varphi(\eta)}\right)^{2(m-n)} \frac{y_{n}(\eta)}{Y_{n}(\eta)},
$$

we see that $\sqrt{3.11}$ follows. The boundedness of $\left\{\max _{k}\left|A_{k, n}^{\eta}\right|\right\}$ is a consequence of 6.30 and 6.13).

\section{Proofs of Theorems 5 and 6}

Proof of Theorem 5. Let $q_{n}$ be the denominators of $\Pi_{n}$. We start by showing that

$$
q_{n}=u_{n-m} q_{n, m}, \quad q_{n, m}=(1+o(1)) q,
$$

locally uniformly in $\mathbb{C} \backslash z(q)$. This follows from [10, Thm. 2.4] in the same fashion as 6.1 followed from [9, Thm. 2.4]. The requirements placed on $\mu$ are the same, so they are satisfied. However, in [10, Thm. 2.4] there are also restrictions placed on the interpolation schemes. Namely, an interpolation scheme $\mathscr{E}$ should be such that $\operatorname{supp}(\mathscr{E}) \cap([c, d] \cup \mathrm{z}(q))=\emptyset$, the probability counting measures of points in $E_{n}$ would converge to some Borel measure with finite logarithmic energy, and the argument functions of polynomials $v_{n}$ would have uniformly bounded derivatives on $[c, d]$.

Clearly, the first two requirement placed on the interpolation scheme is the second requirement of the admissibility property. Hence, we only need to show the uniform boundedness of the derivatives of the arguments of $v_{n}$. Clearly, it amounts to show that

$$
\limsup _{n \rightarrow \infty} \frac{1}{2}\left\|\operatorname{Im}\left(\frac{v_{n}^{\prime}}{v_{n}}\right)\right\|_{[c, d]}=\limsup _{n \rightarrow \infty} \frac{1}{2}\left\|\sum_{e \in E_{n} \cap \mathbb{C}} \operatorname{Im}\left(\frac{1}{\cdot-e}\right)\right\|_{[c, d]}<\infty .
$$

Since $\operatorname{Im}(t-e)=\operatorname{Im}(\bar{e}-t)$ for $t \in[c, d]$, we have that

$$
\begin{aligned}
\left|\sum \operatorname{Im}\left(\frac{1}{t-e}\right)\right| & =\frac{1}{2}\left|\sum \operatorname{Im}\left(\frac{1}{t-\Delta_{n}(e)}\right)-\sum \operatorname{Im}\left(\frac{1}{t-\bar{e}}\right)\right| \\
& =\frac{1}{2}\left|\operatorname{Im}\left(\sum \frac{\Delta_{n}(e)-\bar{e}}{\left(t-\Delta_{n}(e)\right)(t-\bar{e})}\right)\right| \leq \frac{1}{2 s^{2}} \sum\left|\Delta_{n}(e)-\bar{e}\right|,
\end{aligned}
$$

where the sums are taken over $e \in E_{n} \cap \mathbb{C}$ and $s>0$ is such that $|t-e| \geq s$ for all $e \in E_{n}$ and $n \in \mathbb{N}$. So, 7.2 and therefore 7.1 follow from the admissibility of $\mathscr{E}$. 
It is well-known [25, Lem. 6.1.2] and is easily seen from the defining properties of Padé approximants and the Fubini-Tonelli theorem that

$$
\int t^{j} q(t) q_{n}(t) \frac{d \mu(t)}{v_{n}(t)}=0, \quad j=0, \ldots, n-m-1,
$$

and

$$
\left(\mathfrak{f}-\Pi_{n}\right)(z)=\frac{v_{n}(z)}{\left(q_{n} q l_{n-m}\right)(z)} \int \frac{\left(q_{n} q l_{n-m}\right)(t)}{z-t} \frac{d \mu(t)}{v_{n}(t)}, \quad z \in \mathfrak{D}_{\mathfrak{f}},
$$

for any polynomial ${ }_{n-m} l$ of degree at most $n-m$. Now, using decomposition 7.1 and denoting

$$
d \nu_{n}:=\frac{q_{n+m, m} q}{v_{n+m}} d \mu=\frac{q_{n+m, m} q h \hbar \hbar_{\mathbf{x}}}{v_{n+m}} d \omega_{[c, d]},
$$

orthogonality relations (7.3) become

$$
\int t^{j} u_{n}(t) d \nu_{n}(t)=0, \quad j=0, \ldots, n-1
$$

It is also quite easy to see that the asymptotic behavior of $u_{n}$ is governed by Theorem 7 applied with $h_{n}=q_{n+m, m} q$. The orthogonality relation above also imply that

$$
R_{n}(z):=\int \frac{u_{n}(t)}{z-t} d \nu_{n}(t)=\frac{1}{u_{n}(z)} \int \frac{u_{n}^{2}(t)}{z-t} d \nu_{n}(t), \quad z \in \mathfrak{D} .
$$

Thus, putting $l_{n-m}=u_{n-m}$, we can rewrite 7.4 as

$$
\mathfrak{f}-\Pi_{n}=\frac{v_{n} R_{n-m}}{u_{n-m} q_{n, m} q} .
$$

Hence, we derive from 5.3 and 7.1 that

$$
\mathfrak{f}-\Pi_{n}=[1+o(1)] \frac{v_{n} \gamma_{n-m} S_{n-m}^{2}}{\mathfrak{w} q q_{n, m}}=[2+o(1)] G_{\dot{\nu}_{n-m}} S_{\dot{\nu}_{n-m}}^{2} \frac{\psi^{2(n-m)} v_{n}}{\mathfrak{w} q_{n, m} q}
$$

locally uniformly in $\mathfrak{D}$. Therefore, we get from $(2.10)$ and $(7.5)$ that

$$
\begin{aligned}
G_{\dot{\nu}_{n-m}} S_{\dot{\nu}_{n-m}}^{2} \frac{\psi^{2(n-m)} v_{n}}{q_{n, m} q} & =G_{\dot{\mu}} S_{\dot{\mu}}^{2} \frac{G_{q_{n, m}} S_{q_{n, m}}^{2}}{q_{n, m} \psi^{m}} \frac{G_{q} S_{q}^{2}}{q \psi^{m}} \frac{v_{n} \psi^{2 n}}{G_{v_{n}} S_{v_{n}}^{2}} \\
& =\frac{G_{\dot{\mu}} S_{\dot{\mu}}^{2} r_{n}}{r_{m}\left(q_{n, m} ; \cdot\right) r}=[1+o(1)] G_{\dot{\mu}} S_{\dot{\mu}}^{2} \frac{r_{n}}{r^{2}}
\end{aligned}
$$

locally uniformly in $\mathfrak{D}_{\mathfrak{f}}$, where $r_{n}$ and $r$ are defined as in the statement of this theorem. Combining (7.8) with 7.9 we get 4.2 .

Finally, observe that the boundedness of the variation of argument of $h$ was needed in order to appeal to [10, Thm. 2.4]. However, when the rational summand of $\mathfrak{f}$ is not present $(q \equiv 1), 4.2$ is a consequence of Theorem 7 only and the latter does not require the boundedness of the variation of argument of $h$. 
Proof of Theorem 6. The asymptotic equality in 4.3 is exactly the one in (7.1). The fact that $u_{n-m}$ have no zeros on compact sets in $\mathfrak{D}$ follows since the asymptotic behavior of $u_{n-m}$ is governed by Theorem 7 with $\nu_{n}$ given by 7.5 and all the zeros of such orthogonal polynomials approach $[c, d]$.

Let $\eta \in \mathrm{z}(q)$. As in the proof of Theorem 4, we may suppose without loss of generality that $m(\eta)=m$, i.e. $\eta$ is the only zero of $q$. Using the notation of Theorem 7, we can rewrite 7.7 as

$$
\frac{u_{n-m}^{2} q_{n, m} q}{\gamma_{n} v_{n}}\left(\mathfrak{f}-\Pi_{n}\right)=\frac{1}{\gamma_{n-m}} u_{n-m} R_{n-m}=: y_{n},
$$

or equivalently

$$
y_{n}=Y_{n} q_{n, m}+\frac{u_{n-m}\left(\mathfrak{f}_{\mu} q_{n}-p_{n}\right)}{\gamma_{n-m} v_{n}} q, \quad Y_{n}:=\frac{u_{n-m}^{2} p}{\gamma_{n-m} v_{n}},
$$

where $\mathfrak{f}=\mathfrak{f}_{\mu}+p / q$. It follows from $\sqrt{5.3}$ that

$$
y_{n} \mathfrak{w}=1+o(1) \quad \text { locally uniformly in } \mathfrak{D} \text {. }
$$

In particular, it means that sequences $\left\{\left|y_{n}^{(k)}(\eta)\right|\right\}$ are uniformly bounded above and away from zero for all $k \in \mathbb{N}$. Moreover, $(7.10)$ yields that

$$
y_{n}^{(k)}(\eta)=\left(Y_{n} q_{n, m}\right)^{(k)}(\eta), \quad k=0, \ldots, m-1 .
$$

This, for instance, implies that neither of $\eta_{k, n}, k=1, \ldots, m$, the zeros of $q_{n, m}$, is equal to $\eta$. Further, using (5.3), 2.10, and (7.1), we get that

$$
Y_{n}=[1 / 2+o(1)] \psi^{2 m} p /\left(G_{\dot{\mu} q^{2}} S_{\dot{\mu} q^{2}}^{2} r_{n}\right)
$$

uniformly in some neighborhood of $\eta$. Now, it it clear that we may proceed exactly as in the proof of Theorem 4 with the only difference that we set

$$
\left(A_{k, n}^{\eta}\right)^{m}:=\left(1+\delta_{k, n}\right) r_{n}(\eta) / \chi_{n}^{m}=-\left(1+\delta_{k, n}\right)\left(r_{n} y_{n} / Y_{n}\right)(\eta)
$$

\section{Numerical Experiments}

The Hankel operator $\mathcal{H}_{f}$ with symbol $f \in H^{\infty}+C(\mathbb{T})$ is of finite rank if and only if $f$ is a rational function [19, Thm. 3.11]. In practice one can only compute with finite rank operators, due to the necessity of ordering the singular values, so a preliminary rational approximation to $f$ is needed when the latter is not rational. One way to handle this problem is to truncate the Fourier series of $f$ at some high order $N$. This provides us with a rational function $f_{N}$ that approximates $f$ in the Wiener norm which, in particular, dominates any $L^{p}$ norm on the unit circle, $p \in[1, \infty]$. It was proved in [15] that the best approximation operator from $H_{n}^{\infty}$ (mapping $f$ to $g_{n}$ according to $(3.3)$ ) is continuous in the Wiener norm provided $(n+1)$-st singular value of the Hankel operator is simple. It was shown in [2, Cor. 2] that the last assertion is satisfied for Hankel operators with symbols in some open 
dense subset of $H^{\infty}+C(\mathbb{T})$, and the same technique can be used to prove that it is also the case for the particular subclass (1.1). Thus, even though the simplicity of singular values cannot be asserted beforehand, it is generically true. When it prevails, one can approximates $f_{N}$ instead of $f$ and get a close approximation to $g_{n}$ when $N$ is large enough. This amounts to perform the singular value decomposition of $\mathcal{H}_{f_{N}}$ (see [26, Ch. 16]).

As to Padé approximants, we restricted ourselves to the classical case and we constructed their denominators by solving the orthogonality relations 7.3 with $v_{n} \equiv 1$. Thus, finding these denominators amounts to solving a system of linear equations whose coefficients are obtained from the moments of the measure $\mu$.

The following computations were carried with MAPLE 8 software using 35 digits precision. On the figures the solid line stands for the support of the measure and circles denote the poles of the correspondent approximants. The approximated function is given by the formula

$$
\begin{aligned}
\mathfrak{f}(z)= & \int_{[-0.7,0]} \frac{7 e^{i t}}{z-t} \frac{d t}{\sqrt{(t+0.7)(0.4-t)}}+\int_{[0,0.4]} \frac{i t+1}{z-t} \frac{d t}{\sqrt{(t+0.7)(0.4-t)}} \\
& +\frac{1}{5 !(z-0.7-0.2 i)^{6}} .
\end{aligned}
$$
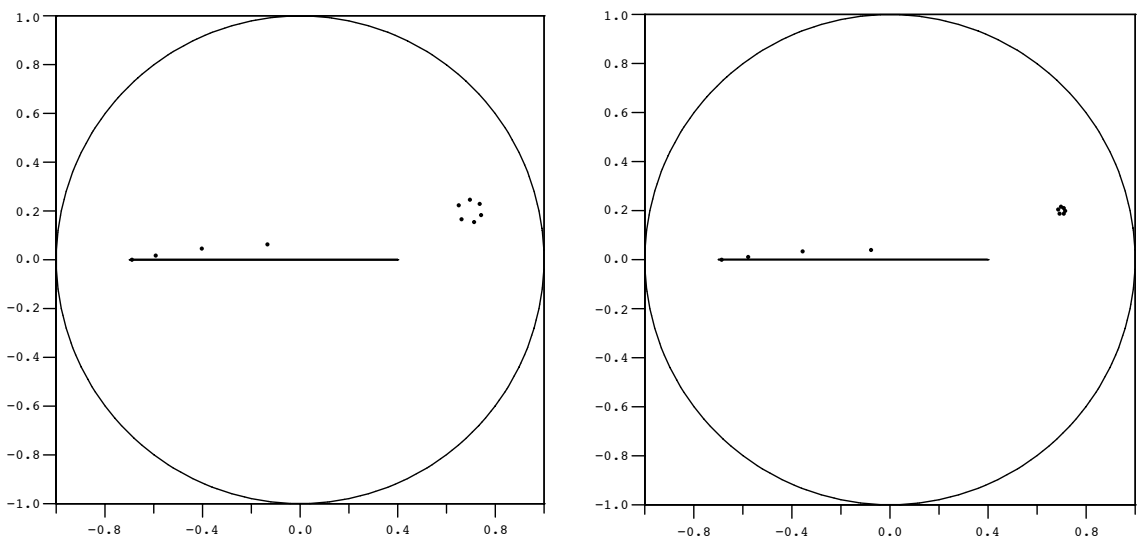

Figure 1. Poles of Padé (left) and AAK (right) approximants of degree 10.

Acknowledgment. I express my sincere gratitude to Dr. L. Baratchart for valuable discussions and comments, his reading the manuscript and suggesting this problem. 

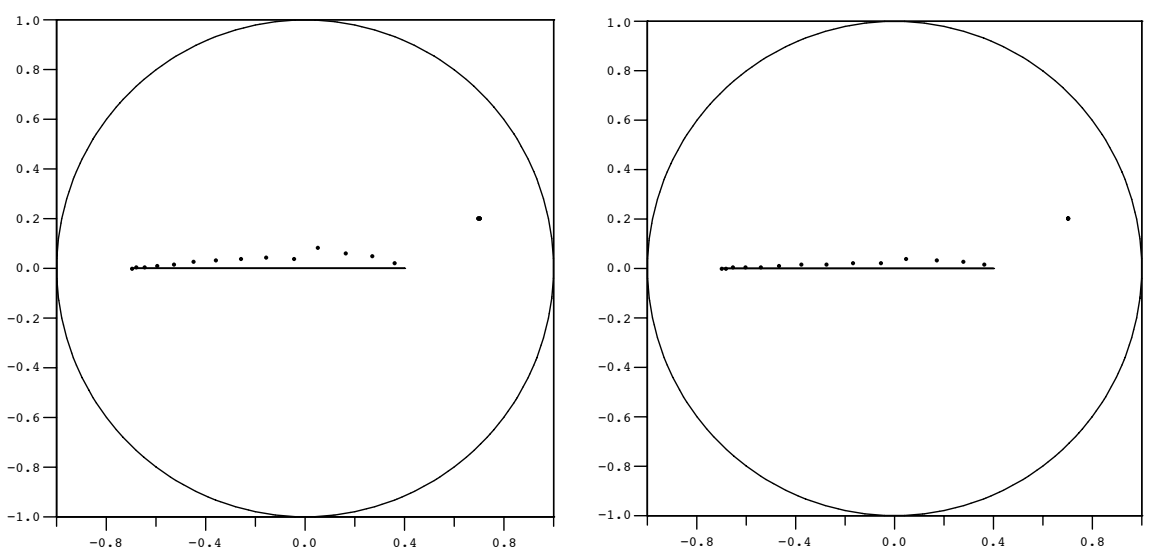

Figure 2. Poles of Padé (left) and AAK (right) approximants of degree 20.
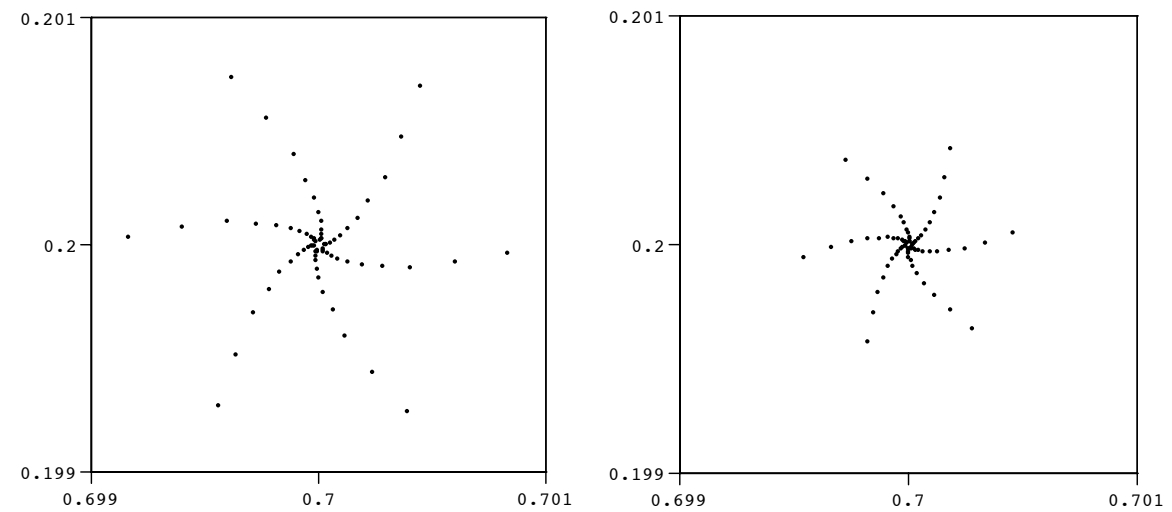

Figure 3. Poles of Padé (left) and AAK (right) approximants of degrees 21-33 lying in an neighborhood of the polar singularity.

\section{References}

[1] V.M. Adamyan, D.Z. Arov, and M.G. Krein. Analytic properties of Schmidt pairs for a Hankel operator on the generalized Schur-Takagi problem. Math. USSR Sb., 15:31-73, 1971.

[2] L. Baratchart, J. Leblond, and J.R. Partington. Problems of Adamyan-Arov-Krein type on subsets of the circle and minimal norm extentions. Constr. Approx., 16(3):333-357, 2000. 
[3] L. Baratchart, F. Mandrèa, E.B. Saff, and F. Wielonsky. 2-D inverse problems for the Laplacian: a meromorphic approximation approach. J. Math. Pures Appl., 86:1-41, 2006.

[4] L. Baratchart, V.A. Prokhorov, and E.B. Saff. Best meromorphic approximation of Markov functions on the unit circle. Found. Comput. Math., 1(4):385-416, 2001.

[5] L. Baratchart and F. Seyfert. An $L^{p}$ analog to AAK theory for $p \geq 2$. J. Funct. Anal., 191(1):52-122, 2002.

[6] L. Baratchart, H. Stahl, and F. Wielonsky. Asymptotic error estimates for $L^{2}$ best rational approximants to Markov functions. J. Approx. Theory, 108(1):53-96, 2001.

[7] L. Baratchart and M. Yattselev. Convergent interpolation to Cauchy integrals over analytic arcs. To appear in Found. Comput. Math., http://arxiv.org/abs/0812.3919.

[8] L. Baratchart and M. Yattselev. Convergent interpolation to Cauchy integrals over analytic arcs of Jacobi-type weights. In preparation.

[9] L. Baratchart and M. Yattselev. Meromorphic approximants to complex Cauchy transforms with polar singularities. To appear in Mat. Sb., http://arxiv.org/abs/0806.4681.

[10] L. Baratchart and M. Yattselev. Multipoint Padé approximants to complex Cauchy transforms with polar singularities. J. Approx. Theory, 2(156):187-211, 2009.

[11] M.S. Derevyagin and V.A. Derkach. On the convergence of Padé approximants for generalized Nevalinna functions. Trans. Moscow Math. Soc., 68:119-162, 2007.

[12] J.B. Garnett. Bounded Analytic Functions, volume 236 of Graduate Texts in Mathematics. Springer, New York, 2007.

[13] A.A. Gonchar. On the convergence of Padé approximants for some classes of meromorphic functions. Mat. Sb., 97(139):607-629, 1975. English transl. in Math. USSR Sb. 26(4):555-575, 1975.

[14] A.A. Gonchar and S.P. Suetin. On Padé approximants of meromorphic functions of Markov type. Current problems in mathematics, 5, 2004. In Russian, available electronically at http://www.mi.ras.ru/spm/pdf/005.pdf.

[15] E. Hayashi, L.N. Trefethen, and M.H. Gutknecht. The CF Table. Constr. Approx., 6(2):195-223, 1990.

[16] A.L. Levin and E.B. Saff. Szegő asymptotics for minimal Blaschke products. In A. A. Gonchar and E. B. Saff, editors, Progress in Approximation Theory, pages 105-126, Springer-Verlag, Berlin/New York, 1992.

[17] A.A. Markov. Deux démonstrations de la convergence de certaines fractions continues. Acta Math., 19:93-104, 1895.

[18] Z. Nehari. Conformal Mapping. International Series in Pure and Applied Mathematics. McGraw-Hill Book Company, Inc., New York, 1952.

[19] J.R. Partington. An Introduction to Hankel operators. Student texts in Maths. Cambridge University Press, Cambridge, UK, 1988.

[20] V.V. Peller. Hankel Operators and Their Applications. Springer Monographs in Mathematics. Springer-Verlag, New York, 2003.

[21] V.A. Prokhorov. On $L^{p}$-generalization of a theorem of Adamyan, Arov, and Krein. J. Approx. Theory, 116(2):380-396, 2002. 
[22] E.A. Rakhmanov. Convergence of diagonal Padé approximants. Mat. Sb., 104(146):271-291, 1977. English transl. in Math. USSR Sb. 33:243-260, 1977.

[23] T. Ransford. Potential Theory in the Complex Plane, volume 28 of London Mathematical Society Student Texts. Cambridge University Press, Cambridge, 1995.

[24] E.B. Saff and V. Totik. Logarithmic Potentials with External Fields, volume 316 of Grundlehren der Math. Wissenschaften. Springer-Verlag, Berlin, 1997.

[25] H. Stahl and V. Totik. General Orthogonal Polynomials, volume 43 of Encycl. Math. Cambridge University Press, Cambridge, 1992.

[26] N.J. Young. An Introduction to Hilbert Space. Cambridge University Press, Cambridge, 1988.

Maxim Yattselev

e-mail: myattsel@sophia.inria.fr

INRIA, Project APICS

2004 route des Lucioles - BP 93

06902 Sophia-Antipolis, France 